\title{
Parametric vertical coordinate formulation for multiscale, Boussinesq, and non-Boussinesq ocean modeling
}

\author{
Y. Tony Song ${ }^{\mathrm{a}, *}$, Thomas Y. Hou ${ }^{\mathrm{b}}$ \\ ${ }^{a}$ Earth and Space Sciences Division, Jet Propulsion Laboratory, California Institute of Technology, \\ Pasadena, CA 91109, USA \\ b Applied and Computational Mathematics, California Institute of Technology, Pasadena, CA 91125, USA
}

Received 6 October 2004; received in revised form 15 December 2004; accepted 10 January 2005

Available online 17 March 2005

\begin{abstract}
Two physical parameters are introduced into the basic ocean equations to generalize numerical ocean models for various vertical coordinate systems and their hybrid features. The two parameters are formulated by combining three techniques: the arbitrary vertical coordinate system of Kasahara [Kasahara, A., 1974. Various vertical coordinate systems used for numerical weather prediction. Mon. Weather Rev. 102, 509-522], the Jacobian pressure gradient formulation of Song [Song, Y.T., 1998. A general pressure gradient formation for ocean models. Part I: Scheme design and diagnostic analysis. Mon. Weather Rev. 126 (12), 3213-3230], and a newly introduced parametric function that permits both Boussinesq (volume-conserving) and non-Boussinesq (mass-conserving) conditions. Based on this new formulation, a generalized modeling approach is proposed. Several representative oceanographic problems with different scales and characteristics - coastal canyon, seamount topography, non-Boussinesq Pacific Ocean with nested eastern Tropics, and a global ocean model — have been used to demonstrate the model's capabilities for multiscale applications. The inclusion of non-Boussinesq physics in the topography-following ocean model does not incur computational expense, but more faithfully represents satellite-observed oceanbottom-pressure data. Such a generalized modeling approach is expected to benefit oceanographers in solving multiscale ocean-related problems by using various coordinate systems on the same numerical platform. (c) 2005 Elsevier Ltd. All rights reserved.
\end{abstract}

\footnotetext{
* Corresponding author. Tel.: +1 818393 4876; fax: +1 8183936720 .

E-mail address: song@pacific.jpl.nasa.gov (Y.T. Song).
} 
Keywords: Generalized vertical coordinate system; Non-Boussinesq; Multiscale applications

\section{Introduction}

Numerical ocean models have become multi-disciplinary research tools for use in a variety of ocean-related areas, including coastal ocean predictions, climate simulations, data assimilations, and coupled physical-biogeochemical studies. Several ocean models have been developed in the last few decades, including the Bryan-Cox-Type models (MOM, Bryan, 1969; Cox, 1984), the Princeton Ocean Model (POM, Blumberg and Mellor, 1987), the Miami Isopycnic Coordinate Ocean Model (MICOM, Bleck et al., 1992), the MIT general circulation model (MITgcm, Marshall et al., 1997), the S-Coordinate Rutgers University Model (SCRUM, Song and Haidvogel, 1994), and the Regional Ocean Model System (ROMS, Haidvogel et al., 2000; Shchepetkin and McWilliams, in press). As noted by various model-comparison studies such as DYNAMO (DYNAMO Group, 1997), DYMEE (Chassignet et al., 2000), and two recent reviews on this subject by Haidvogel and Beckmann (1999) and by Griffies et al. (2000), the choice of a vertical coordinate system is the single most important aspect of an ocean model's design. In fact, the existing ocean circulation models can be roughly divided into four classes, each centering around a different way of discretizing the vertical coordinate, based on the $z$ (Cartesian)-coordinate, $\sigma$ (terrainfollowing)-coordinate, $\rho$ (isopycnal)-coordinate, or $p$ (pressure)-coordinate system.

(1) z-coordinate system. This is the simplest and most commonly used vertical coordinate system, which involves discretizing a vertical column of the ocean into fixed levels. The vertical levels may have different thicknesses and great advantages for resolving surface mixing and upper ocean dynamics. However, difficulties have been encountered in representing varying topography, especially near coastal boundaries where the ocean bottom rapidly varies from shallow to deep water. In such cases, the $z$-coordinates have had to approximate the bottom slope as a series of staircases. This approximation was found to cause an inaccurate representation of bottom kinematic conditions (Gerdes, 1993), exchanging masses between shallow and deep waters (Roberts and Wood, 1997; Beckmann and Döscher, 1997), and bottom pressure torques (Bell, 1999; Hughes and De Cuevas, 2001). Recently, progress has been made to overcome the topography representation problem by partial or shaved-cell techniques (Adcroft et al., 1997; Pacanowski and Ghanadesikan, 1998). Such a technique has also been used in the Hamburg Ocean Primitive Equation (HOPE) model (Drijfhout et al., 1996) and the Parallel Ocean Program (POP) model (Smith et al., 1992).

(2) $\sigma$-coordinate system. This is another widely used vertical coordinate system that transforms the irregular physical domain, bounded by free surface and varying bottom topography, into a regular computational domain. It is commonly used in estuarine and coastal ocean studies (Oey et al., 1985). The major advantage of this system is that it follows the topography, and therefore naturally represents the bottom kinematic and stress boundary conditions (Gerdes, 1993) and bottom pressure torques (Song and Wright, 1998). A corresponding disadvantage is that it cannot maintain equally high resolution near the surface layer independent of local depth. This problem is minimized via a stretching function, commonly called the s-coordinate (Song and Haidvogel, 1994), to maintain resolution in both surface and bottom mixed layers away from coasts. Another well-known problem is that the terrain-following coordinates may generate pressure gradient 
errors over steep topography (Haney, 1991). Progress has also been made to reduce the errors by both second-order Jacobian schemes (e.g., Song, 1998) and higher-order schemes (e.g., Chu and Fan, 1997). More recently, Shchepetkin and McWilliams (2003) proposed to modify the Jacobian schemes by fitting the density or pressure profiles into polynomials and then using analytical integration to further reduce the pressure gradient errors.

(3) $\rho$-coordinate system. This is an advanced approach of discretizing the vertical coordinate system by dividing the water column into layers of constant density. This system is more natural for large-scale ocean circulation because water tends to move mostly parallel to density surfaces (Bleck et al., 1992). However, it does not easily handle nonlinear equations of state, and resolution degrades when density stratification is weak (Haidvogel and Beckmann, 1998). It also suffers from similar problems with the resolution of steep topography as with the level-models, because isopycnals do not generally conform to topographic variations. However, a piecewise linear fashion of topographic representation has been used to avoid the need to distinguish bottom from sides (Bleck and Benjamin, 1993) as traditionally done with $z$-models (Griffies et al., 2000).

(4) p-coordinate system. To emphasize the importance of the compressible (conserving mass) flow of ocean waters, the pressure coordinate system of Phillips (1957) is also used to formulate ocean models (Huang et al., 2001). In such a system, the mass, instead of the volume of the ocean model, is conserved and the bottom pressure becomes a prognostic variable, which provides advantages for assimilating ocean-bottom-pressure measurements (Song and Zlotnicki, 2003). So far, this system has not been widely used in realistic applications. Difficulties in dealing with topography or pressure gradient calculation cannot be avoided if using either fixed pressure or terrain-following levels.

Clearly, these traditional vertical coordinate systems are not, by themselves, optimal everywhere in the ocean. Different model classes have different advantages/disadvantages for solving different scales of problems (Haidvogel and Beckmann, 1999; Griffies et al., 2000). As ocean models have become multi-disciplinary research tools, a variety of applications from coastal to global scale and from physical to biogeochemical problems require numerical models to be flexible and highly optimized. Global ocean modeling cannot ignore estuarine and coastal oceans; while coastal regions are not independent from open oceans (Walsh, 1991). The limitations imposed by the traditional coordinate systems should be minimized, if possible, to allow easy communication among those model classes and to extend their capabilities for multiscale applications.

Indeed, some efforts have been undertaken to improve the single-coordinate deficiencies in each of those model classes. Most of these efforts have been trying to extend an individual coordinate system to have some hybrid features of other coordinate systems. For example, Gerdes (1993) proposed the hybrid $z-\sigma$ coordinate system for the GFDL model. Bottom boundary layer schemes are also tested in the $z$-coordinate models (Killworth and Edwards, 1999; Song and Chao, 2000). In the terrain-following model class, a great deal of energy has also been focused on reducing pressure gradient errors, including the work of Beckmann and Haidvogel (1993), Song (1998), Chu and Fan (1997), and Shchepetkin and McWilliams (2003). More recently, Mellor et al. (2001) implemented the $z$-coordinate system into POM as an option. Pietrzak et al. (2002) tested the hybrid $z-\sigma$ technique. In the isopycnal model class, HYCOM (Bleck, 2002) uses hybrid $\rho-z$ coordinates, an advanced version of MICOM. To consider mass-conserving properties, relaxations of Boussinesq approximations have also been tested (e.g., Greatbatch 
et al., 2001; Losch et al., 2004). Despite these early efforts, each model class still has its intrinsic limitations for certain types of applications. Each hybrid model has to adopt its inherent model structure. Some of the hybrid schemes may lead to discontinuity or a mismatch at the interface of two different coordinate regions (Deleersnijder and Seekers, 1992).

The present work focuses on generalizing the model coordinate system for multiscale, Boussinesq (volume-conserving) and non-Boussinesq (mass-conserving) flow applications. The idea of using generalized vertical coordinates for atmosphere and ocean models emerged several decades ago. Pioneer work includes Kasahara (1974), Bleck (1978), Simmons and Burridge (1981), Baer and Ji (1989), Zhu et al. (1992), and Konor and Arakawa (1997) for atmospheric modeling, and Bleck and Boudra (1981) and Gerdes (1993) for ocean modeling. Different from those approaches, Song and Haidvogel (1994) proposed the $s$-coordinate system with variable parameters to adjust computational levels for better resolutions in the surface and bottom layers without altering the original terrain-following numerical schemes. However, the $s$-coordinate system does not permit non-Boussinesq physics, nor align the computational levels with isopycnals in the interior of the ocean. The methodology of this study differs from previous studies by introducing two physical parameters into the basic ocean equations to further generalize an ocean model for using the optimal or hybrid features of those traditional coordinate systems. The two parameters are formulated by combining three techniques: the arbitrary vertical coordinate system of Kasahara (1974), the Jacobian pressure gradient formulation of Song (1998), and a newly introduced parametric function that permits both Boussinesq and non-Boussinesq conditions. It will be shown that the introduction of the two parameters does not incur computational expense, but allows users to choose multiple vertical structures for multiscale applications on the same model platform.

This paper is organized into three parts: model formulation, numerical method, and demonstrations. The numerical implementation will be based on an existing model platform (SCRUM/ ROMS); however, it is not limited by the specific model configuration. The demonstrations consist of solving several representing oceanographic problems with different scales and characteristics: coastal canyon, seamount topography, a nested regional model into a non-Boussinesq Pacific Ocean model, and a global ocean model. These application problems are chosen to test model capabilities of coupling large-scale ocean dynamics with topography-following feature for coastal oceans.

\section{Model formulation}

\subsection{The basic equations}

We begin with the basic ocean equations in the Cartesian coordinate system with the $z$-axis pointing vertically upwards and the $(x, y)$-plane occupying the undisturbed water surface. The horizontal momentum equations are written in the form:

$$
\frac{\partial u}{\partial t}+\vec{v} \cdot \nabla u-f v=-\frac{1}{\rho} \frac{\partial p}{\partial x}+\frac{1}{\rho} \frac{\partial p}{\partial z}\left(\rho K_{M} \frac{\partial u}{\partial z}\right)+\mathscr{D}_{u}+\mathscr{F}_{u}
$$

and 


$$
\frac{\partial v}{\partial t}+\vec{v} \cdot \nabla v-f u=-\frac{1}{\rho} \frac{\partial p}{\partial y}+\frac{1}{\rho} \frac{\partial p}{\partial z}\left(\rho K_{M} \frac{\partial v}{\partial z}\right)+\mathscr{D}_{v}+\mathscr{F}_{v} .
$$

The tracer (temperature, salinity, biological, or chemical component) equations can likewise be written:

$$
\frac{\partial \hat{T}}{\partial t}+\vec{v} \cdot \nabla \hat{T}=\frac{1}{\rho} \frac{\partial}{\partial z}\left(\rho K_{H} \frac{\partial \hat{T}}{\partial z}\right)+\mathscr{D}_{\hat{T}}+\mathscr{F}_{\hat{T}} .
$$

The hydrostatic balance equation is

$$
\frac{\partial p}{\partial z}=-g \rho(T, S, p) .
$$

Finally, the mass continuity equation is

$$
\frac{\partial \rho}{\partial t}+\frac{\partial \rho u}{\partial x}+\frac{\partial \rho v}{\partial y}+\frac{\partial \rho w}{\partial z}=0
$$

The notations used in these equations are as follows:

$u, v, w$ the $x, y, z$ components of vector velocity $\vec{v}$

$\nabla \quad$ the three-dimensional gradient operator

$\hat{T}(x, y, z, t)$ tracers (temperature, salinity, or biogeochemical components)

$\rho=\rho_{0}+\rho^{\prime}(x, y, z, t)$ total density

$p \quad$ pressure

$f=2 \Omega \sin \psi$ Coriolis parameter due to Earth's rotation

$g \quad$ acceleration due to gravity

$K_{M}(x, y, z, t)$ vertical eddy viscosity

$K_{H}(x, y, z, t)$ vertical eddy diffusivity

$\mathscr{D}_{u}, \mathscr{D}_{v}, \mathscr{D}_{\hat{T}}$ horizontal viscous and diffusive terms

$\mathscr{F}_{u}, \mathscr{F}_{v}, \mathscr{F}_{\hat{T}}$ forcing, sink, or source terms

Please notice that we have used $\hat{T}$ to represent a tracer component, including temperature $T$ and salinity $S$. Discussions on the vertical eddy coefficients and horizontal viscous and diffusive terms are referred to Song and Haidvogel (1994). In this work the $K$-profile parameterization (KPP) scheme of Large et al. (1994) is used for vertical mixing and rotated tensors of Laplacian and biharmonic formulation are used for horizontal mixing.

Historically, most existing ocean models ( $z$ - and $\sigma$-models) impose incompressible (Boussinesq) approximations on the oceanic flow (Boussinesq, 1903) by (1) using volume conservation to replace mass conservation in the continuity equation, i.e., $\nabla \cdot \vec{v} \approx 0$; (2) using mean density to replace variable density in the momentum equations, i.e., $\frac{1}{\rho} \cdot \nabla p \approx \frac{1}{\rho_{0}} \cdot \nabla p$; and (3) using geopotential depth to replace the pressure in the equation of state, i.e., $\rho(T, S, p) \approx \rho\left(T, S, p_{0}(z)\right)$. Although the effects of such approximations on the model simulation of the ocean circulation remain unclear (Mellor and Ezer, 1995; Huang et al., 2001), it is important to have ocean models represent the real ocean as accurately as possible. Several ways of relaxing the Boussinesq approximations are proposed recently, mainly for $z$-level models (De Szoeke and Samelson, 2002; Greatbatch et al., 2001; Losch et al., 2004). Here we offer an alternative way to relaxing the 
approximations by simply changing one of the two introduced parameters (the following section), which is applicable to any coordinate system. As will be shown later, the inclusion of the compressible physics to the model does not incur computational expense with our parametric coordinate formulation. Such extended model capabilities are needed in currently used community ocean models to faithfully represent satellite sensing data. For example, the TOPEX sea surface elevation represents heat storage of sea water $(\mathrm{Fu}, 1983)$ due to expansion/contraction. The Gravity Recovery and Climate Experiment (GRACE, Tapley et al., 2004) measures ocean bottom pressure anomaly due to mass changes of ocean water (Hughes et al., 2000). It is important to have the ocean model conserving mass, instead of volume, in assimilating satellite sensing data (Huang et al., 2001; Song and Zlotnicki, 2003).

\subsection{The parametric vertical coordinate system}

There are two important reasons for generalizing the vertical coordinate system in ocean models. First, the computation of oceanic flow involves complex boundaries (free-surface, varying coastline, and bottom topography) that do not coincide with the commonly used Cartesian coordinates in physical space. For finite difference methods, the imposition of boundary conditions for such problems has required a complicated interpolation of the data on local grid lines and, typically, a local loss of accuracy. The use of coordinate transformation can avoid some of these problems by mapping an irregular physical space into a regular computational domain (Fletcher, 1991). Second, oceanic flow has far different dynamic scales between its horizontal and vertical directions (Lardner and Song, 1991). Special care should be made on choosing the vertical coordinates and computational levels (Gerdes, 1993). For these reasons, we require our generalized coordinate system to be able to resolve the dynamical structures of the underlying physical solution and to follow the horizontal boundaries as well as possible.

We consider a generalized coordinate system, represented by

$$
\xi=\xi(x, y), \quad \eta=\eta(x, y), \quad s=s(x, y, z, t)
$$

where $s$ represents the vertical transformation, $\xi$ and $\eta$ are the horizontal transformations, and $t$ is time. As the focus of the work is on the vertical direction, the horizontal transformation is simplified to the case of time-independent, orthogonal curvilinear coordinates. We also require the coordinate transformation to satisfy tow conditions: (i) a unique, single-valued relationship between $(x, y, z)$ and $(\xi, \eta, s)$ at any given time $t$, particularly, a vertical monotonic relationship is assumed for $z$ as a function of $s$

$$
z=z(\xi, \eta, s, t), \quad-1 \leqslant s \leqslant 0
$$

and (ii) the derivatives of all the mapping functions are continuous. With these conditions, the coordinate metric tensor can be written as

$$
(\mathrm{d} c)_{\xi}=\left(\frac{1}{m}\right) \mathrm{d} \xi, \quad(\mathrm{d} c)_{\eta}=\left(\frac{1}{n}\right) \mathrm{d} \eta, \quad(\mathrm{d} z)_{s}=\left(H_{z}\right) \mathrm{d} s
$$

where $m(\xi, \eta)$ and $n(\xi, \eta)$ are the scale factors which relate the differential distances $(\Delta \xi, \Delta \eta)$ to the actual (physical) arc length $\mathrm{d} c$ (Fletcher, 1991), and $H_{z}=\frac{\partial z}{\partial s}$ is the vertical metric factor corresponding to a uniform grid $\Delta s$. One of the commonly used coordinate systems is the spherical 
coordinates for many ocean models, in which $\xi=\lambda$ and $\eta=\psi$ represent longitude and latitude, and $u$ and $v$ the zonal and meridional components of velocity. The transformation metrics are

$$
\frac{1}{m}=a \cos \psi, \quad \frac{1}{n}=a, \quad H_{z}=\left(\zeta+h_{\mathrm{c}}\right)+\left(h(x, y)-h_{\mathrm{c}}\right) C^{\prime}(s)
$$

where $a$ is the radius of the Earth, $\zeta$ is the sea-surface height, $h(x, y)$ is the bathymetry, $h_{\mathrm{c}}$ is a constant chosen to be the minimum depth of the bathymetry or the width of boundary layer, and $C^{\prime}(s)$ is the derivative of the stretching function, introduced by Song and Haidvogel (1994),

$$
C(s)=(1-b) \frac{\sinh (\theta s)}{\sinh \theta}+b \frac{\tanh [\theta(s+0.5)]-\tanh (0.5 \theta)}{2 \tanh (0.5 \theta)}
$$

and $\theta$ and $b$ are the surface and bottom control parameters. Typical parameter ranges are $0 \leqslant \theta \leqslant 20$ and $\theta \leqslant b \leqslant 1$, respectively. Such an analytical stretching function (called $s$-coordinates) has been used in SCRUM and ROMS worldwide. In the following, we will further generalize the vertical coordinate system.

Using the relationships (2.6)-(2.8) and following Kasahara (1974) and Song (1998), the basic ocean equations (2.1)-(2.5) can be written into a new form of "differential-integral" equations (notice the changes in the pressure and vertical diffusion terms):

$$
\begin{aligned}
& \frac{\partial}{\partial t}\left(\frac{\phi u}{m n}\right)+\frac{\partial}{\partial \xi}\left(\frac{\phi u u}{n}\right)+\frac{\partial}{\partial \eta}\left(\frac{\phi u v}{m}\right)+\frac{\partial}{\partial s}\left(\frac{\phi \Omega u}{m n}\right) \\
&= \frac{\phi}{n B_{z}} \int_{s}^{0}\left\{\frac{\partial z}{\partial s^{\prime}} \frac{\partial b}{\partial \xi}-\frac{\partial z}{\partial \xi} \frac{\partial b}{\partial s^{\prime}}\right\} \mathrm{d} s^{\prime}+\frac{\partial}{\partial s}\left(\frac{B_{z}^{2} K_{M}}{\phi m n} \frac{\partial u}{\partial s}\right) \\
&+\left\{\frac{f}{m n}+v \frac{\partial}{\partial \xi}\left(\frac{1}{n}\right)-u \frac{\partial}{\partial \eta}\left(\frac{1}{m}\right)\right\} \phi v+\frac{\phi}{m n}\left\{\mathscr{D}_{u}+\mathscr{F}_{u}\right\} \\
& \frac{\partial}{\partial t}\left(\frac{\phi v}{m n}\right)+\frac{\partial}{\partial \xi}\left(\frac{\phi u v}{n}\right)+\frac{\partial}{\partial \eta}\left(\frac{\phi v v}{m}\right)+\frac{\partial}{\partial s}\left(\frac{\phi \Omega v}{m n}\right) \\
&=\frac{\phi}{m B_{z}} \int_{s}^{0}\left\{\frac{\partial z}{\partial s^{\prime}} \frac{\partial b}{\partial \eta}-\frac{\partial z}{\partial \eta} \frac{\partial b}{\partial s^{\prime}}\right\} \mathrm{d} s^{\prime}+\frac{\partial}{\partial s}\left(\frac{B_{z}^{2} K_{M}}{\phi m n} \frac{\partial v}{\partial s}\right) \\
& \quad-\left\{\frac{f}{m n}+v \frac{\partial}{\partial \xi}\left(\frac{1}{n}\right)-u \frac{\partial}{\partial \eta}\left(\frac{1}{m}\right)\right\} \phi u+\frac{\phi}{m n}\left\{\mathscr{D}_{v}+\mathscr{F}_{v}\right\} \\
& \frac{\partial}{\partial t}\left(\frac{\phi \hat{T}}{m n}\right)+\frac{\partial}{\partial \xi}\left(\frac{\phi u \hat{T}}{n}\right)+\frac{\partial}{\partial \eta}\left(\frac{\phi v \hat{T}}{m}\right)+\frac{\partial}{\partial s}\left(\frac{\phi \Omega \hat{T}}{m n}\right) \\
&=\frac{\partial}{\partial s}\left(\frac{B_{z}^{2} K_{H}}{\phi m n} \frac{\partial \hat{T}}{\partial s}\right)+\frac{\phi}{m n}\left\{\mathscr{D}_{\hat{T}}+\mathscr{F}_{\hat{T}}\right\}
\end{aligned}
$$

and

$$
\frac{\partial}{\partial t}\left(\frac{\phi}{m n}\right)+\frac{\partial}{\partial \xi}\left(\frac{\phi u}{n}\right)+\frac{\partial}{\partial \eta}\left(\frac{\phi v}{m}\right)+\frac{\partial}{\partial s}\left(\frac{\phi \Omega}{m n}\right)=0
$$


where $\Omega$ is the vertical velocity in the generalized coordinate system, defined by

$$
\Omega=\frac{\partial s}{\partial z}\left\{w-\left(\frac{\partial z}{\partial t}\right)_{s}-u m \frac{\partial z}{\partial \xi}-v n \frac{\partial z}{\partial \eta}\right\}
$$

$b=-g \rho / \rho_{0}$ is the buoyancy, the vertical height can be obtained by

$$
z=\int_{-1}^{s} \frac{\phi}{B_{z}} \mathrm{~d} s^{\prime}-h
$$

In the new basic ocean equations we have introduced the parametric function as

$$
\phi(\xi, \eta, s, t)=H_{z}(\xi, \eta, s, t) B_{z}(\xi, \eta, s, t)
$$

where $H_{z}$ and $B_{z}$ are the newly defined two physical parameters: the metric parameter and the Boussinesq parameter, respectively. Their forms can be written as $H_{z}=\frac{\partial z}{\partial s}$ and $B_{z}=\frac{\rho}{\rho_{0}}$ in deriving the generalized coordinate equations (2.11)-(2.15) from the primitive equations (2.1)-(2.5). However, they can be any positive, monotonic functions (vertically) as will be explained later. One of the examples is assuming $B_{z}=1$ in Eqs. (2.11)-(2.15) to recover the commonly used Boussinesq primitive equations. It can be shown that $\left(H_{z}, B_{z}\right)$ is a set of parametric curves of variable $\mathrm{s}$ at a given location $(\xi, \eta)$ and time $t$.

In addition, Eqs. (2.11)-(2.15) are subject to boundary conditions on sea surface $\zeta(\xi, \eta, t)$ and at ocean bottom $h(\xi, \eta)$. It should be pointed out that the formulation of boundary conditions is not independent from the choice of vertical coordinate systems. For example, the traditional $z$-models may need staircase or shaved-cell approximations of topography, therefore, have different formulations with those topography-following models. A generic derivation of the boundary conditions is given by Kasahara (1974). Here, we only give the boundary conditions for the coordinate systems that follow free surface and bottom topography, but the model interior can be any system. In such cases, the surface conditions, evaluated at $z=\zeta($ or $s=0)$, are

$$
\begin{aligned}
& \frac{B_{z}^{2} K_{M}}{\phi} \frac{\partial u}{\partial s}=\tau_{s}^{\xi}(\xi, \eta, t) \\
& \frac{B_{z}^{2} K_{M}}{\phi} \frac{\partial v}{\partial s}=\tau_{s}^{\eta}(\xi, \eta, t) \\
& \frac{B_{z}^{2} K_{H}}{\phi} \frac{\partial T}{\partial s}=\frac{Q_{T}}{\rho_{0} C_{p}}+\frac{1}{\rho_{0} C_{p}} \frac{\mathrm{d} Q_{T}}{\mathrm{~d} T}\left(T-T_{\mathrm{ref}}\right) \\
& \frac{B_{z}^{2} K_{H}}{\phi} \frac{\partial S}{\partial s}=\frac{(e-p) S}{\rho_{0}}
\end{aligned}
$$

where $\tau_{s}^{\xi}$ and $\tau_{s}^{\eta}$ are the components of wind stress acting on the free surface in the $\xi$ and $\eta$ directions, respectively, $Q_{T}$ is the heat fluxes, $e$ and $p$ are the evaporation and precipitation rates, respectively, and $C_{p}$ is the heat capacity of sea water. (Note: the salinity flux should be modified accordingly for non-Boussinesq cases.) Correspondingly, at the sea bed, $z=-h$ (or $s=-1$ ), the boundary conditions are 


$$
\begin{aligned}
& \frac{B_{z}^{2} K_{M}}{\phi} \frac{\partial u}{\partial s}=\tau_{\mathrm{b}}^{\xi}(\xi, \eta, t) \\
& \frac{B_{z}^{2} K_{M}}{\phi} \frac{\partial v}{\partial s}=\tau_{\mathrm{b}}^{\eta}(\xi, \eta, t) \\
& \frac{B_{z}^{2} K_{H}}{\phi} \frac{\partial T}{\partial s}=0 \\
& \frac{B_{z}^{2} K_{H}}{\phi} \frac{\partial S}{\partial s}=0
\end{aligned}
$$

where $\tau_{\mathrm{b}}^{\xi}=\left(\gamma_{1}+\gamma_{2} \sqrt{u^{2}+v^{2}}\right) u$ and $\tau_{\mathrm{b}}^{\eta}=\left(\gamma_{1}+\gamma_{2} \sqrt{u^{2}+v^{2}}\right) v$, and $\gamma_{1}$ and $\gamma_{2}$ are coefficients of linear and quadratic bottom friction, respectively. It should be noted that this combined linear and quadratic formulation of the bottom drag has the flexibility for both shallow or deep ocean applications. For deep ocean, if only the linear bottom drag is needed, $\gamma_{2}$ can be set to zero. Similarly, $\gamma_{1}$ can be set to zero for shallow seas.

It is worthy pointing out that there are several differences from the $s$-coordinate model of Song and Haidvogel (1994): (1) a Boussinesq factor is introduced in the basic ocean equations as well as in the boundary conditions, (2) the parametric function $H_{z}$ becomes adaptive, instead of analytical, and (3) the Jacobian form in the pressure gradient terms is required to allow parametric coordinate transformations. Notice that we have combined both horizontal and vertical transformations into one step in deriving the parametric equations. The horizontal curvilinear coordinates $\xi=\xi(x, y)$ and $\eta=\eta(x, y)$ are useful for resolving coastlines in regional ocean models. For those readers only interested in the vertical transformation, the horizontal transformations can be ignored by setting $m=n=1$ in the above equations.

\subsection{The generalized modeling approach}

Here we explain how the two parameters, $H_{z}$ and $B_{z}$, can be used to develop a generalized ocean model for multiscale applications. First of all, let us show how they can be simplified to recover some of the known traditional and hybrid coordinate systems. The reader is suggested to substitute the following functions $(\mathrm{A}-\mathrm{F})$ into Eqs. (2.11)-(2.15) for verification:

(A) To become a hybrid $z-\sigma$-level model, the two parameters can be simply chosen as

$$
\left.\begin{array}{l}
H_{z}=\Delta z \\
B_{z}=1
\end{array}\right\} \Rightarrow \phi=\Delta z .
$$

In this case, the Boussinesq parameter $B_{z}$ is constant and inactive. The parametric function $\phi$ depends on the choice of the vertical grid $\Delta z$, which is independent from topographic variations in the interior of the ocean, but allows to align with the topography in bottom layer. If $\phi=\Delta z$ is imposed for the whole water column, the ocean bottom topography has to be approximated step-like by the fixed vertical levels, which becomes the traditional $z$-level model. Such a capability has been demonstrated in POM by Mellor et al. (2001) and will not be discussed here. 
(B) To become the traditional $\sigma$-coordinate model, they can be chosen as

$$
\left.\begin{array}{l}
H_{z}=H \\
B_{z}=1
\end{array}\right\} \Rightarrow \phi=H
$$

where $H=\zeta+h$ is the total water depth. In such a case our Eqs. (2.11)-(2.15) should become the original equations of Blumberg and Mellor (1987).

(C) To become a hybrid isopycnal system, the parameters can be chosen as

$$
\left.\begin{array}{l}
H_{z}=-\frac{1}{g \rho} \frac{\partial p}{\partial s} \\
B_{z}=\frac{\rho}{\rho_{0}}
\end{array}\right\} \Rightarrow \phi=-\frac{1}{g \rho_{0}} \frac{\partial p}{\partial s} .
$$

In this case, the computational levels follow isopycnals in the interior of the ocean, but gradually align with the free-surface and topography in the surface and bottom layer, respectively. This technique can be used to avoid problems of topographic truncations and surface layer outcrops of isopycnals (Bleck and Benjamin, 1993).

(D) To use the traditional pressure coordinate system of Phillips (1957), the two parameters become

$$
\left.\begin{array}{l}
H_{z}=\frac{p_{\mathrm{bs}}}{g \rho} \\
B_{z}=\frac{\rho}{\rho_{0}}
\end{array}\right\} \Rightarrow \phi=\frac{p_{\mathrm{bs}}}{g \rho_{0}}
$$

where $p_{\mathrm{bs}}=p_{\mathrm{b}}-p_{\mathrm{s}}, p_{\mathrm{s}}$ and $p_{\mathrm{b}}$ are the surface and bottom pressure, respectively, and the pressuresigma coordinate system is $p=p_{\mathrm{s}}-p_{\mathrm{bs}} s$ and $-1 \leqslant s \leqslant 0$. Such a pressure coordinate system has been used by Huang et al. (2001).

(E) To become the s-coordinate system of Song and Haidvogel (1994), we obtain

$$
\left.\begin{array}{l}
H_{z}=\left(\zeta+h_{\mathrm{c}}\right)+\left(h-h_{\mathrm{c}}\right) C^{\prime}(s) \\
B_{z}=1
\end{array}\right\} \Rightarrow \phi=H_{z}
$$

It can be verified that Eqs. (2.11)-(2.15) with (2.30) are equivalent to those equations of Song and Haidvogel (1994).

(F) Finally, we introduce a new non-linear pressure-coordinate system, called stretching pressure coordinates or $s p$-coordinates for short, as

$$
p=p_{s} \alpha(s)+p_{\mathrm{c}} \beta(s)+p_{\mathrm{b}} \gamma(s), \quad-1 \leqslant s \leqslant 0
$$

where $p_{\mathrm{c}}$ is a constant pressure near the thermocline, and $\alpha(s), \beta(s)$, and $\gamma(s)$ are stretching functions determined by users. The two parameters in Eqs. (2.11)-(2.15) become

$$
\left.\begin{array}{l}
H_{z}=-\frac{1}{g \rho} H_{p} \\
B_{z}=\frac{\rho}{\rho_{0}}
\end{array}\right\} \Rightarrow \phi=\frac{1}{g \rho_{0}} H_{p}
$$

where $H_{p}=\left(p_{\mathrm{c}}-p_{\mathrm{s}}\right)+\left(p_{\mathrm{b}}-p_{\mathrm{c}}\right) C^{\prime}(s)$. It should be noted that this system has advantages over the traditional $p$-coordinates used in atmospheric modeling (Phillips, 1957) for better resolving surface and bottom layers of the ocean.

In summary, above formulations demonstrate that each of the known traditional or hybrid vertical coordinate systems is a particular case of Eqs. (2.11)-(2.15), differing by the choice of the two 


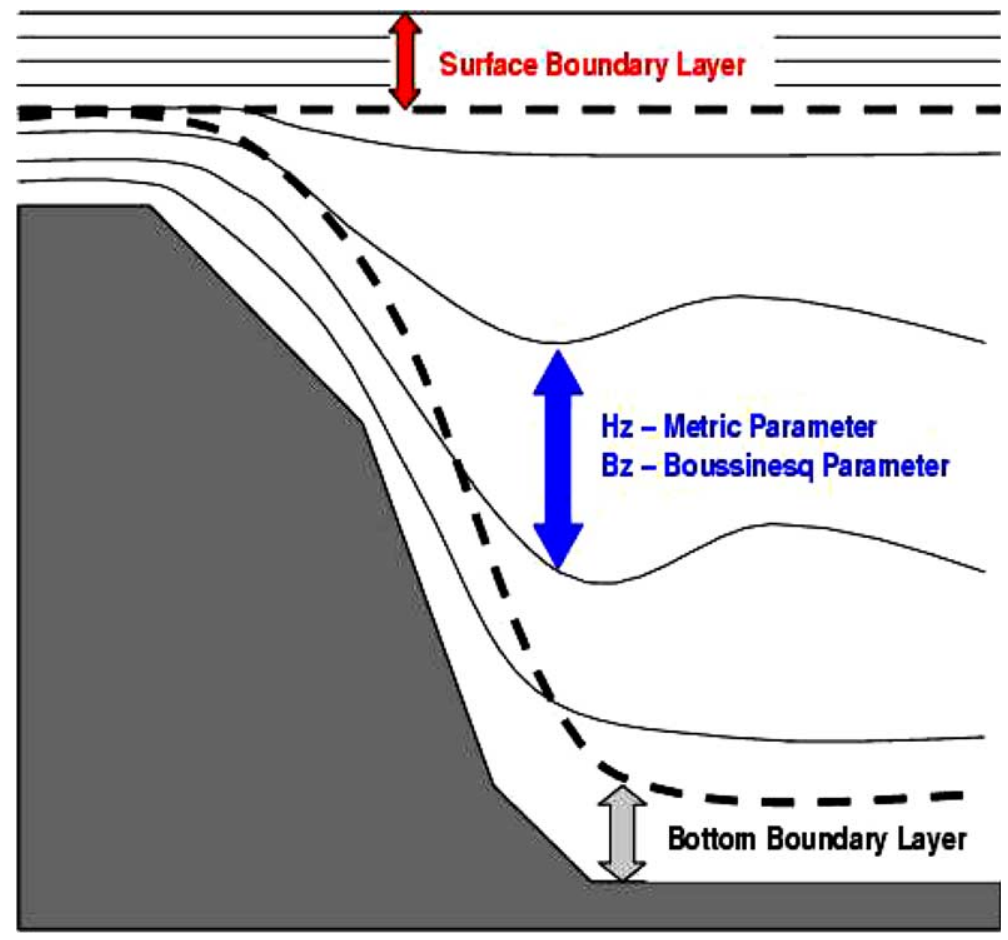

Fig. 1. Schematic of the proposed parametric modeling approach: the metric parameter $H_{z}$, is the height of the computational layers, allowing to follow isopycnals, bottom topography, or geopotential levels. The Boussinesq parameter $B_{z}$ is the mass to volume ratio for determining compressible conditions of the computational layer. The computational levels (solid lines) allow to align with free surface and bottom topography gradually within the boundary layers (marked by the dashed lines). In realistic applications, the highest possible vertical resolution in the surface and bottom layers as well as in the coastal oceans is desired to achieve accurate representation of the dynamic processes of air-sea and ocean-Earth interactions.

parameters, as indicated in Fig. 1 schematically. These two parameters have physical meanings. The metric parameter, $H_{z}$, is the height of the computational layers, allowing to follow isopycnals, bottom topography, or geopotential levels. If $H_{z}$ is restricted to constant $z$-levels, $\rho$-levels, or $p$-levels as in the traditional models, a staircase approximation of topography is the consequence. The Boussinesq parameter, $B_{z}$, is the mass to volume ratio for determining Boussinesq conditions (compressibility on incompressibility) of the computational layer. If the Boussinesq approximation is assumed as in the above cases (A), (B), and (E), the volume of that layer is conserved. On the other hand, if non-Boussinesq approximation is assumed as in the above cases (C), (D), and $(\mathrm{F})$, the mass of the layer should be conserved.

Theoretically the two parameters can be arbitrarily chosen as long as they satisfy the monotonic conditions

$$
\Delta z \leqslant H_{z} \leqslant \frac{\partial z}{\partial s} \Delta s, \quad 1 \leqslant B_{z} \leqslant \frac{\rho}{\rho_{0}}
$$

in the dimension of $s$, where $\frac{\partial z}{\partial s} \geqslant 1$ and $\rho / \rho_{0} \geqslant 1$ are assumed to be physical meaningful. Mathematically the choices of model vertical systems only have two freedoms, controlled by the two 
parameters. In fact, all above six cases are special cases of (2.33). The Boussinesq approximation is the extreme case when $B_{z}=1$. Therefore, the model can be designed to have generalized modeling capabilities and to offer users great freedom for choosing desired model structures by choosing these two parameters, instead of changing the numerical algorithms. The imposed monotonic condition on the parametric function is to avoid collapsing of computational levels, whether they are allowed to intersect with topography and sea-surface is the user's choice. In the next two sections, we will present the numerical method and its applications for solving multiscale, Boussinesq and non-Boussinesq problems.

\section{Numerical method}

Here we briefly describe the numerical method used in implementing the proposed parametric coordinate system. The method, although similar to Song and Haidvogel (1994), has been modified along the years of development of SCRUM/ROMS (http://marine.rutgers.edu/po/models/). To be consistent with the new parametric coordinate system proposed here, some of the numerical schemes have also been modified accordingly. Therefore, it is necessary to give the readers the exact numerical configuration used in this study.

\subsection{The mode splitting technique}

Our model is based on the staggered (Arakawa C-grid) finite volume configuration. In the staggered grid the oceanic flow is discretized into many control volumes. Each of them has six surfaces with distances $\Delta \xi, \Delta \eta$ and $\Delta s$ in the general coordinate system (2.6)-(2.8). The pressure $\left(p_{i, j, k}\right)$ and tracers $\left(\hat{T}_{i j k}\right)$ located at the center of the control volume, and the velocity components $\left(u_{i}, v_{j}, w_{k}\right.$; $\left.u_{i+1}, v_{j+1}, w_{k+1}\right)$ are placed at the centers of the surfaces.

The staggered grid has two major advantages. First, it has good conservation (of mass, etc.) properties (Fletcher, 1991). The conservation of physical properties in ocean models is critical for long-term climate simulations and equilibrium circulation of coastal currents. Since errors in the discrete equations cannot be eliminated completely, certain integral properties of the continuous equations should be satisfied to avoid the gradual development of large errors (Arakawa and Suarez, 1983). Song and Wright (1998) also emphasized that the pressure gradient force should be treated carefully to conserve total energy, momentum, and bottom torque. Second, the finite volume method allows complicated computational domains to be discretized in a simpler way than either the isoparametric finite element formulation or generalized curvilinear coordinates. This method does not depend on the mesh regularity, but is suited to approximate mixed derivatives and degenerates into the finite difference method when the mesh is regular (Hermeline, 2000). This is particularly important for ocean modeling because complex geometry and varying topography are common features. However, the staggered grid needs averaging two neighbor points to compute a variable of interest in a control volume. Such an averaging process can be

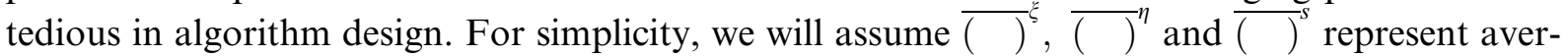
ages taken over the distances $\Delta \xi, \Delta \eta$ and $\Delta s$, respectively.

Based on the finite volume scheme, we solve the ocean basic equations (2.11)-(2.15) separately for their external mode representing the depth-averaged flow and the internal mode 
representing the total flow. The main reason for splitting these two modes is to satisfy the CFL condition arising from the fast moving surface gravity waves. Such a splitting technique has been widely used in many three-dimensional atmosphere and ocean circulation models (e.g., Arakawa and Lamb, 1977; Blumberg and Mellor, 1987). The semi-discrete (spatial only) form for the internal mode is

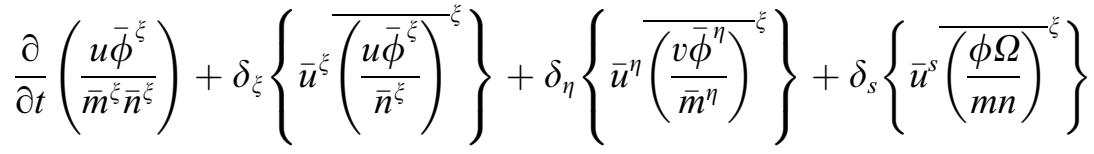

$$
\begin{aligned}
& -\left\{\frac{f}{m n}+\bar{v}^{\eta} \delta_{\xi} \overline{\left(\frac{1}{n}\right)^{\xi}}-\bar{u}^{\xi} \delta_{\eta} \overline{\left(\frac{1}{m}\right)^{\eta}}\right\} \phi \bar{v}^{\eta}
\end{aligned}
$$

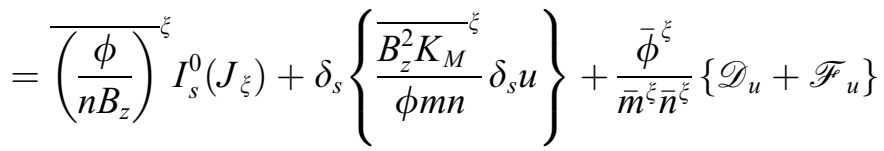

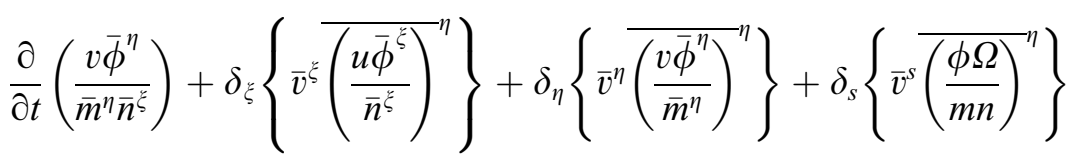

$$
\begin{aligned}
& +\left\{\frac{f}{m n}+\bar{v}^{\eta} \delta_{\xi} \overline{\left(\frac{1}{n}\right)^{\xi}}-\bar{u}^{\xi} \delta_{\eta} \overline{\left(\frac{1}{m}\right)^{\eta}}\right\} \phi \bar{u}^{\xi}
\end{aligned}
$$

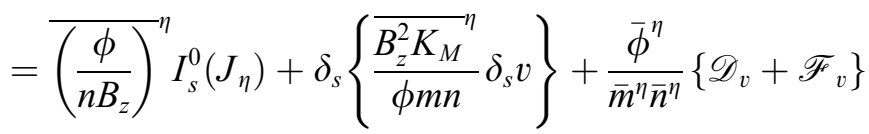

$$
\begin{aligned}
& \frac{\partial}{\partial t}\left(\frac{\phi \hat{T}}{m n}\right)+\delta_{\xi}\left(\frac{u \bar{\phi}^{\xi} \overline{\hat{T}}^{\xi}}{\bar{n}^{\xi}}\right)+\delta_{\eta}\left(\frac{v \bar{\phi}^{\eta} \overline{\hat{T}}^{\eta}}{\bar{m}^{\eta}}\right)+\delta_{s}\left(\frac{\Omega \phi \overline{\hat{T}}^{s}}{m n}\right)=\frac{\phi}{m n}\left\{\mathscr{D}_{\hat{T}}+\mathscr{F}_{\hat{T}}\right\} \\
& \frac{\partial}{\partial t}\left(\frac{\phi}{m n}\right)+\delta_{\xi}\left(\frac{u \bar{\phi}^{\xi}}{\bar{n}^{\xi}}\right)+\delta_{\eta}\left(\frac{v \bar{\phi}^{\eta}}{\bar{m}^{\eta}}\right)+\delta_{s}\left(\frac{\Omega \phi}{m n}\right)=0 .
\end{aligned}
$$

Here, $\delta_{\xi}, \delta_{\eta}$ and $\delta_{s}$ denote simple centered finite-volume approximations to $\partial / \partial \xi, \partial / \partial \eta$ and $\partial / \partial s$ with the differences taken over the distances $\Delta \xi, \Delta \eta$ and $\Delta s$, respectively. $I_{s}^{0}$ indicates a secondorder vertical integral computed as a sum from level $s$ to the surface at $s=0$. The pressure gradients are written into Jacobian form as given by Song (1998)

$$
J_{\xi}(b, z)=\frac{\partial z}{\partial s} \frac{\partial b}{\partial \xi}-\frac{\partial z}{\partial \xi} \frac{\partial b}{\partial s}, \quad J_{\eta}(b, z)=\frac{\partial z}{\partial s} \frac{\partial b}{\partial \eta}-\frac{\partial z}{\partial \eta} \frac{\partial b}{\partial s} .
$$

The external mode equations can be obtained by vertical integration of the momentum equations $(2.11,2.12)$ and the mass (or volume) continuity equation (2.14). Using the corresponding surface and bottom conditions, we obtain the depth-averaged equations in the semi-discrete form: 


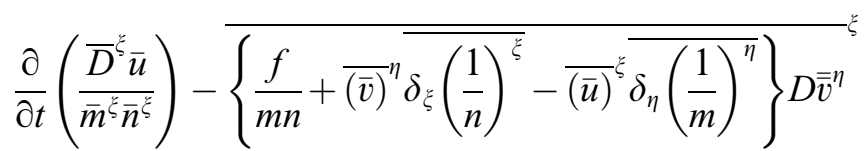

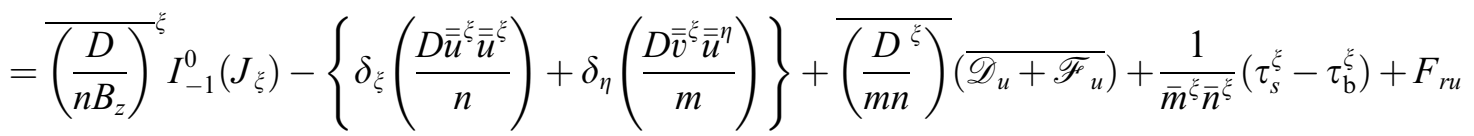

$$
\begin{aligned}
& \frac{\partial}{\partial t}\left(\frac{\bar{D}^{\eta} \bar{v}}{\bar{m}^{\eta} \bar{n}^{\eta}}\right)-\overline{\left\{\frac{f}{m n}+\overline{(\bar{v})^{\eta}} \delta_{\xi}\left(\frac{1}{n}\right)^{\xi}\right.}-\overline{\left.(\bar{u})^{\xi} \delta_{\eta}\left(\frac{1}{m}\right)^{\eta}\right\} D \overline{\bar{v}}^{\xi}} \\
& \quad=\overline{\left(\frac{D}{n B_{z}}\right)^{\eta}} I_{-1}^{0}\left(J_{\eta}\right)-\left\{\delta_{\xi}\left(\frac{D \overline{\bar{u}}^{\eta} \bar{v}^{\xi}}{n}\right)+\delta_{\eta}\left(\frac{D \overline{\bar{v}}^{\eta} \overline{\bar{v}}^{\eta}}{m}\right)\right\}+\overline{\frac{D}{m n}}^{\eta}\left(\overline{\mathscr{D}_{v}+\mathscr{F}_{v}}\right)+\frac{1}{\bar{m}^{\eta} \bar{n}^{\eta}}\left(\tau_{s}^{\eta}-\tau_{\mathrm{b}}^{\eta}\right)+F_{r v}
\end{aligned}
$$

and

$$
\frac{\partial}{\partial t}\left(\frac{D}{m n}\right)+\delta_{\xi}\left(\frac{\bar{D}^{\xi} \bar{u}}{\bar{n}^{\xi}}\right)+\delta_{\eta}\left(\frac{\bar{D}^{\eta} \bar{v}}{\bar{m}^{\eta}}\right)=0
$$

here $D=\int_{-1}^{0} \phi d$ is the vertically integrated mass, and $\bar{u}$ and $\bar{v}$ are the depth-average velocity for Boussinesq cases and mass-averaged velocity for non-Boussinesq cases. Notice that $\overline{\bar{u}}^{\eta}=(\overline{\bar{u}}){ }^{\eta}$.

It should be pointed out that the depth-averaged equations (external mode) are not independent of the vertically varying equations (internal mode); they are coupled through the nonlinear and pressure gradient terms, which are represented by $F_{r u}$ and $F_{r v}$. The coupling procedures can be important for the model performance. We will discuss the coupling issue after introducing the time stepping schemes.

\subsection{The implicit scheme for the internal mode}

Because ocean flow has very different horizontal and vertical length scales, differing by one or two orders of magnitude, they are usually solved by different numerical schemes (Lardner and Song, 1991). Implicit treatment of the vertical parts of the process is necessary because time-step limitation is more severe in the vertical than in the horizontal directions. Here, we follow the method of Song and Haidvogel (1994), using the implicit generalized Crank-Nicolson scheme for the vertical direction and the third-order Adams-Bashforth (AB3) scheme for the horizontal directions. In this method, the vertical parts of the process can be written as:

$$
\frac{\partial(\phi U)}{\partial t}-\frac{\partial}{\partial s}\left(\frac{B_{z}^{2} \kappa}{\phi} \frac{\partial U}{\partial s}\right)=m n R_{U}
$$

where $R_{U}$ represents all of the forcing terms other than the vertical viscosity or diffusion. We use $U$ as the generic variable for Eqs. ((3.1)-(3.3)) and introduce 


$$
\widetilde{U}=\lambda U^{n+1}+(1-\lambda) U^{n}
$$

where $\lambda$ is an implicitness parameter, and approximate equation (3.1) and boundary conditions (2.18) and (2.22) as

$$
\begin{aligned}
& \frac{\phi U^{n+1}-\phi U^{n}}{\Delta t}-\frac{\partial}{\partial s}\left(\frac{B_{z}^{2} K}{\phi} \frac{\partial \widetilde{U}}{\partial s}\right)=m n \widetilde{R}_{U} \\
& B_{z}^{2} K \frac{\partial \widetilde{U}}{\partial s}=\phi \tilde{\tau}_{s}, \quad B_{z}^{2} K \frac{\partial \widetilde{U}}{\partial s}=\phi \tilde{\tau}_{\mathrm{b}}
\end{aligned}
$$

where $\tilde{\tau}_{s}$ and $\tilde{\tau}_{\mathrm{b}}$ are the surface and bottom boundary conditions. When $\lambda=\frac{1}{2}$, it becomes the trapezoidal scheme. $K$ stands for the corresponding vertical eddy coefficient. On the right hand side of the equation, $\widetilde{R}_{U}$ is evaluated explicitly by the third-order Adams-Bashforth (AB3) scheme

$$
\widetilde{R}_{U}=\frac{1}{12}\left(23 R^{n}-16 R^{n-1}+5 R^{n-2}\right)
$$

Treating $\widetilde{U}$ as the new unknown, we can rewrite Eq. (3.11) as

$$
\frac{\phi \widetilde{U}}{\lambda \Delta t}-\frac{\partial}{\partial s}\left(\frac{B_{z}^{2} K}{\phi} \frac{\partial \widetilde{U}}{\partial s}\right)=\frac{\phi U^{n}}{\lambda \Delta t}+m n \widetilde{R}_{U} .
$$

Once $\widetilde{U}$ is solved, $U^{n+1}$ can be updated through Eq. (3.10). This can be reorganized so that all the terms involving $\widetilde{U}$ are the solutions of the tridiagonal system:

$$
-A_{k} \widetilde{U}_{k-1}+B_{k} \widetilde{U}_{k}-C_{k} \widetilde{U}_{k+1}=D_{k}, \quad k=0, \ldots, N .
$$

The tridiagonal system can be straightforwardly solved in $O(N)$ operations by forward elimination

$$
B_{k}=B_{k}-A_{k} C_{k-1} / B_{k-1}, \quad D_{k}=D_{k}+A_{k} D_{k-1} / B_{k-1}, \quad k=1, \ldots, N
$$

and back substitution

$$
\widetilde{U}_{N}=D_{N} / B_{N}, \quad \widetilde{U}_{k}=\left(D_{k}+C_{k} \widetilde{U}_{k+1}\right) / B_{k}, \quad k=N-1, \ldots, 0 .
$$

Here the coefficients of the tridiagonal system are as follows:

For $k=1, \ldots, N-1$,

$$
\begin{aligned}
A_{k} & =-\left(\frac{B_{z}^{2} K}{\phi}\right)_{k-\frac{1}{2}} \\
B_{k} & =A_{k}+C_{k}+\frac{\phi_{k}}{\lambda \Delta t} \\
C_{k} & =-\left(\frac{B_{z}^{2} K}{\phi}\right)_{k+\frac{1}{2}}
\end{aligned}
$$




$$
D_{k}=m n \Delta s \widetilde{R}_{U k}+\frac{\phi_{k}}{\lambda \Delta t} U_{k}^{n-1}
$$

for $k=0$,

$$
\begin{aligned}
& A_{0}=0 \\
& B_{0}=C_{0}+\frac{\phi_{1}}{\lambda \Delta t} \\
& C_{0}=-\left(\frac{B_{z}^{2} K}{\phi}\right)_{\frac{1}{2}} \\
& D_{0}=m n \Delta s \widetilde{R}_{U 1}-\tilde{\tau}_{\mathrm{b}}
\end{aligned}
$$

and for $k=N$,

$$
\begin{aligned}
& A_{N}=-\left(\frac{B_{z}^{2} K}{\phi}\right)_{N-\frac{1}{2}} \\
& B_{N}=A_{N}+\frac{\phi_{N-1}}{\lambda \Delta t} \\
& C_{N}=0 \\
& D_{N}=m n \Delta s \widetilde{R}_{U N-1}+\tilde{\tau}_{s} .
\end{aligned}
$$

The implicit treatment of the vertical direction is necessary to relax the severe CFL restriction which arises from the very thin vertical grid size near the surface and bottom layers (Fig. 1). In realistic ocean modeling, the highest possible vertical resolution in the surface and bottom layers as well as in the coastal oceans is desired to achieve an accurate representation of the dynamic processes of air-sea and ocean-Earth interactions in the ocean model. Clearly, the longer the time-step is allowed in solving the internal mode equations, the more efficient the model becomes, since the main cost is solving the three-dimensional equations. This is particularly true when more tracers are needed in including biology and chemical components.

However, the time-splitting between the external and internal modes can be a problem if they deviate from each other too much in time stepping. The external mode equations are two dimensional and shorter time-steps are used to resolve the fast moving surface waves. In our model, a leapfrog-trapezoidal (second-order) scheme is used for the external mode. The ratio of the internal time-step to the external one is about 40; therefore, the external mode only has one chance to couple with the internal mode in every 40 external time-steps. Another issue is the accuracy balanced between these two modes. If both modes are solved by the same second-order scheme with a truncation error of $O\left[(\Delta t)^{2}\right]$, they would have different accuracies because one time-step is much (40 times) longer than the other one. Their coupling would degrade the accuracy of the overall solution by the longer time-step scheme. 
To balance the accuracy, Song and Haidvogel (1994) first proposed the third-order Adams-Bashforth (AB3) scheme for the internal mode equations, which is one-order higher than the second-order scheme used for the external mode. This is different from those previous ocean models. For example, the Bryan-Cox model originally used the leapfrog scheme with a Robert filter. Blumberg and Mellor (1987) use the leapfrog-trapezoidal scheme for the external mode and leapfrog-Asselin scheme for internal mode equations. MICOM/HYCOM and POSEIDON (Schopf and Loughe, 1995) use a leapfrog with the time filter for both baroclinic and tracers, and HIM (Hallberg, 2000) uses the predictor-corrector for the baroclinic mode (Griffies et al., 2000). In the traditional second-order schemes, the viscous/diffusive terms have to be treated by an Euler method to avoid unconditional instability (Bryan, 1969), thereby reducing the accuracy to first-order of truncation error. Here, the AB3 can achieve third-order accuracy for all right-hand-side terms in the internal mode equations and reduce the time-splitting oscillation usually associated with the leapfrog scheme.

Recently, Shchepetkin and McWilliams (in press) further investigated the time stepping schemes. Their study confirms that AB3 and LF-AM3 (leapfrog-third-order Adams-Moulton) offer the best accuracy per computational cost, leaving RK4 (Runge-Kutta fourth-order) and leapfrog-trapezoidal schemes behind. In addition, they also proposed to couple the two modes by a weighted-averaging approach for the external time-steps at each internal time-step, which has been used here.

\section{Multiscale applications}

In this section, several representative oceanographic problems have been used to demonstrate the model's capabilities for multiscale applications and test its numerical consistency with multiple coordinate systems. These problems are chosen because of their different scales and characteristics. Traditionally they would have to be solved by different models in order to take advantage of various coordinate systems. Here we show that they can be solved by the new model in the same numerical framework, and those advantages can be explored by simply choosing the two physical parameters. We shall start with a small-scale coastal problem and end with a large-scale global model. In the first two idealized problems, benchmark solutions are known and quantitative comparisons are given. In the other two realistic applications, we focus on evaluating model performances in long-term simulations.

\subsection{The coastal canyon problem}

This problem is designed to compare numerical ocean models in resolving residual (time-mean) currents driven by oscillatory winds over a continental shelf and slope in the presence of an across-shelf canyon (Haidvogel and Beckmann, 1998). The computational geometry is a coastal channel periodic in the $x$ direction, bounded by two inshore and offshore walls. The channel dimensions are $L_{x}=128 \mathrm{~km}$ and $L_{y}=96 \mathrm{~km}$. A grid size of $2 \mathrm{~km}$ is used in both horizontal directions. The topography is a steep continental shelf increasing in depth with cross channel distance intersected by an isolated and idealized canyon. The problem has been configured to offer a wide range of challenges to the ocean models due to the steep topography. The analytical form of the topography is 


$$
h(x, y)=20+1990\left(1+\tanh \left(\left(y-Y_{\mathrm{c}}\right) / L_{\mathrm{s}}\right)\right)
$$

where $Y_{\mathrm{c}}=Y_{0}-L_{\mathrm{c}} \sin ^{24}\left(\pi x / L_{x}\right)$ and $Y_{0}=32 \mathrm{~km}, L_{\mathrm{s}}=10 \mathrm{~km}, L_{\mathrm{c}}=16 \mathrm{~km}$. The circulation in the coastal channel is driven by an along-shore wind stress:

$$
\tau^{x}=\tau_{0}\left(1-\tanh \left(\left(y-\frac{1}{2} L_{y}\right) / L_{\mathrm{w}}\right)\right) / 2
$$

with a sinusoidally varying amplitude of $\tau_{0}=10^{-4} \sin \left(2 \pi t / T_{\mathrm{w}}\right) \mathrm{Pa} \mathrm{kg}^{-1} \mathrm{~m}^{3}$ where $L_{\mathrm{w}}=10 \mathrm{~km}$ and $T_{\mathrm{w}}=10$ days. Constant Coriolis parameter $f=1 \times 10^{-4} \mathrm{~s}^{-1}$ and linear bottom stress coefficient $\gamma_{\mathrm{B}}=3 \times 10^{-4} \mathrm{~m} \mathrm{~s}^{-1}$ are used. Homogeneous fluid flow is assumed. Both the wind and bottom stresses are applied as body forces acting on the whole water column. Laplacian friction with a coefficient $5 \mathrm{~m}^{2} \mathrm{~s}^{-1}$ is used. Vertical eddy viscosity coefficient is $10^{-4} \mathrm{~m}^{2} \mathrm{~s}^{-1}$. Only free-slip lateral conditions are tested here.

We have solved this problem with three different coordinate systems: the original $\sigma$-coordinates, the $s$-coordinates with $\theta=3$ and $b=1$, and the hybrid $\sigma-z$-coordinates. All three experiments

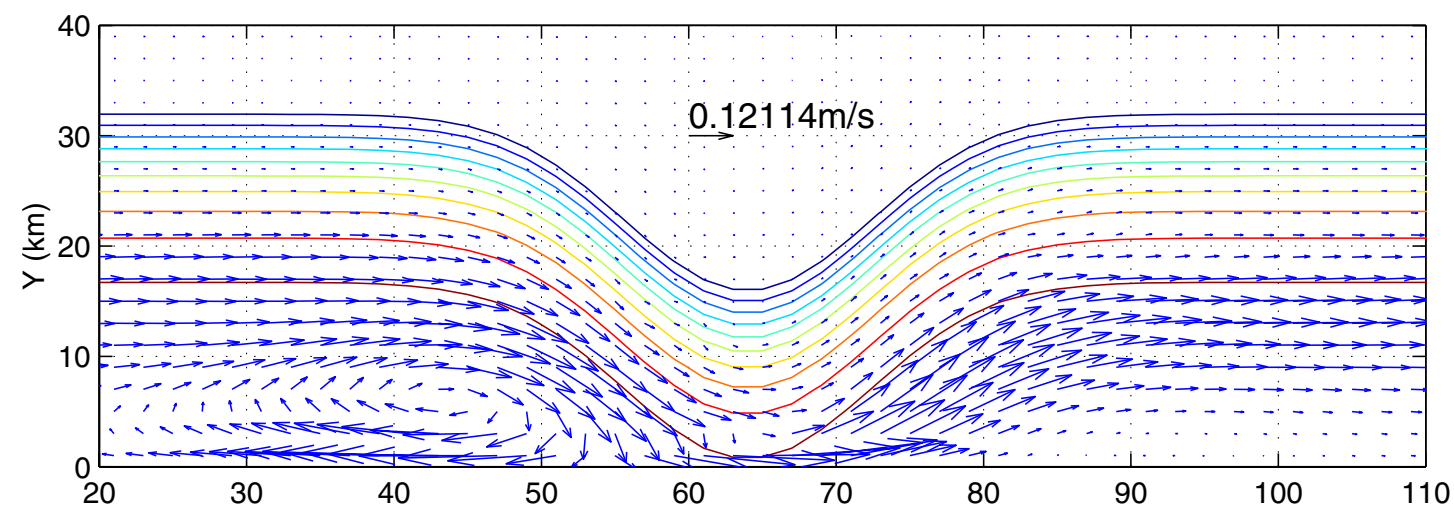

(a)

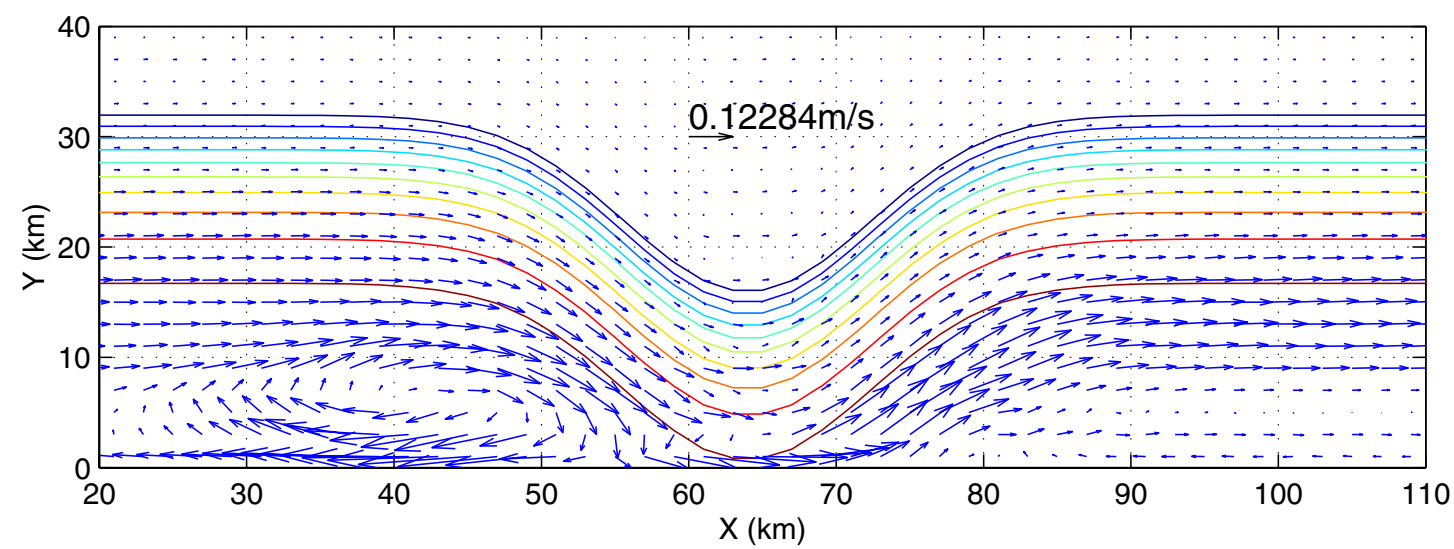

(b)

Fig. 2. Time mean surface currents (a) from the $\sigma$-coordinates and (b) from the hybrid $\sigma-z$ coordinates. Results are averaged from day 90 to day 120 . Contours are the coastal canyon bathymetry. 
have been carried out to day 120 ; the last 30 days ( $90 \leqslant t \leqslant 120$ days) are averaged to give the time-mean (residual) fields. Fig. 2 gives the surface layer velocity over the last 30 days (equivalent to three oscillation periods) from the $\sigma$ and the hybrid $\sigma-z$ experiments. All experiments show the similar features of an anticyclonic eddy at the left side of the canyon and a mean current over the slope. However, some differences can be seen near the coast and from their maximum velocity, which are $0.121 \mathrm{~m} \mathrm{~s}^{-1}$ and $0.123 \mathrm{~m} \mathrm{~s}^{-1}$, respectively. We have also calculated the along-channel, depth- and time-averaged residuals $\bar{u}^{x z t}$ in Fig. 3. The $\sigma$ - and $s$-coordinates give almost the same results, with a maximum of residual $3.95 \mathrm{~cm} \mathrm{~s}^{-1}$, and the hybrid $s-z$ run gives $3.36 \mathrm{~cm} \mathrm{~s}^{-1}$. The net transport through the channel $\overline{h u}^{x z t}$ is about $0.328 \mathrm{~Sv}, 0.331 \mathrm{~Sv}$, and $0.419 \mathrm{~Sv}$, respectively. These results compare well with those (see Table 1) from Haidvogel and Beckmann (1998) and Sheng et al. (1998). Their benchmark solutions are $\bar{u}_{\max }^{x z t}=4.1 \mathrm{~cm} \mathrm{~s}^{-1}$ and $\overline{h u}_{\max }^{x z t}=0.309 \mathrm{~Sv}$, generated by a high-order spectral element model of Iskandarani et al. (1995). It is worthy noting that the partial or shaved-cell techniques (Adcroft et al., 1997) were not available with $z$-models in Haidvogel and Beckmann's collective comparisons, in which $z$-coordinate models performed poorly in resolving the canyon topography. Although the hybrid approach in this example is

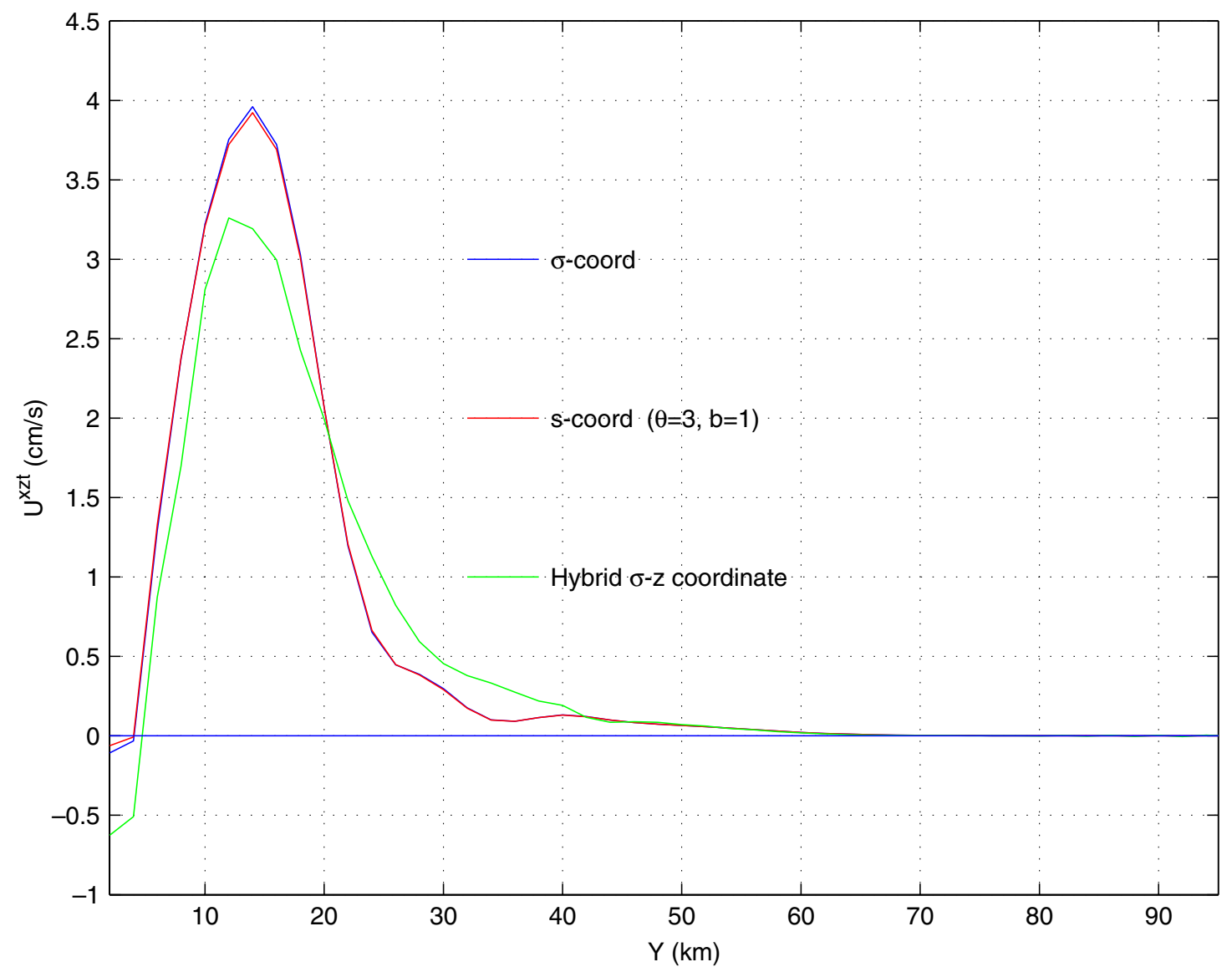

Fig. 3. Intercomparisons of the along-channel, depth- and time-averaged residuals $\bar{U}^{x z t}$. Notice that the $\sigma$ - and $s$ coordinates give almost the same result, which are special cases of our generalized model. 
Table 1

Intercomparison of the homogeneous coastal canyon experiments (free-slip)

\begin{tabular}{llllllll}
\hline Other models & $\bar{u}_{\max }^{t}$ & $\bar{u}_{\max }^{x z t}$ & $\overline{h u}_{\max }^{x z t}(\mathrm{~Sv})$ & Present model & $\bar{u}_{\max }^{t}$ & $\bar{u}_{\max }^{x z t}$ & $\overline{h u}_{\max }^{x z t}(\mathrm{~Sv})$ \\
\hline CANDIE & 12.9 & 3.7 & 0.340 & $\sigma$-coord & 12.1 & 3.95 & 0.328 \\
MICOM & 10.8 & 3.0 & 0.169 & $s$-coord $(\theta=3, b=1)$ & 12.1 & 3.96 & 0.331 \\
SCRUM & 11.9 & 3.6 & 0.264 & Hybrid $\sigma-z$ & 12.3 & 3.36 & 0.419 \\
SEOM & 14.6 & 4.1 & 0.309 & & & & \\
\hline
\end{tabular}

Units are $\mathrm{cm} \mathrm{s}^{-1}$ for velocity and $\mathrm{Sv}\left(1 \mathrm{~Sv}=10^{6} \mathrm{~m}^{3} \mathrm{~s}^{-1}\right)$ for transport.

not as good as the true $\sigma$-model, it is better than the previously reported $z$-level models. The current $z$-models may have improved since then. Nevertheless, our hybrid $\sigma-z$ approach provides an alternative way to tackle such a coastal problem with $z$-levels for open oceans.

\subsection{The seamount topography}

The seamount problem provides a measure of the errors in the pressure gradient term in the presence of steep topography, which have been widely used to test ocean models (Beckmann and Haidvogel, 1993; Song and Wright, 1998; Ezer et al., 2002; Shchepetkin and McWilliams, 2003). Here we show how the pressure gradient error can be reduced by allowing the computational levels to follow $z$-levels or isopycnals in the interior and gradually align with topography in the bottom layer and $z$-levels in the surface layer.

The configuration is a tall, steep seamount in a channel, closed to the north and south and recirculating through the east and west boundaries. The computational domain has dimensions of approximately $320 \mathrm{~km}$ by $320 \mathrm{~km}$, and is divided into $48 \times 48$ grid cells. The isolated seamount is located in the center of the channel and its topography is described by a Gaussian profile

$$
h(x, y)=5000-4500 \mathrm{e}^{-(r / l)^{2}}
$$

where $r=\sqrt{\left(x-x_{\mathrm{c}}\right)^{2}+\left(y-y_{\mathrm{c}}\right)^{2}}$ is the distance from the center of the domain and $l=40 \mathrm{~km}$ is the width of the seamount. The stratification of the ocean is initialized by a single tracer

$$
T(z)=15+3 \mathrm{e}^{z / 500} .
$$

A horizontal Laplacian viscosity is set to $50 \mathrm{~m}^{2} \mathrm{~s}^{-1}$. This is similar to the configuration of Shchepetkin and McWilliams (2003), but slightly different from that of Beckmann and Haidvogel (1993), and Song and Wright (1998) in removing a mean background density and adding a small density perturbation. Although the removal of a mean background density is a common practice in terrain-following coordinate modeling, we will not apply the removal option in the test problems.

We have carried out four experiments: the traditional $\sigma$-coordinates, the $s$-coordinates with $\theta=3$ and $b=0$, the hybrid $s-z$, and the hybrid $s-\rho$ coordinates based on the background density for the Boussinesq parameter. All experiments have 11 vertical levels: the $\sigma$-levels are evenly

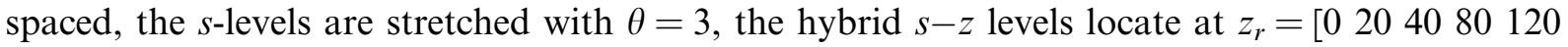
260800160028004000 5000] meters from deep ocean to the $s$-levels near the seamount, and the hybrid $s-\rho$ levels are $\rho\left(z_{r}\right)$ from the deep ocean to the $s p$-levels near the seamount. The model starts from a state of rest with no surface forcing. Theoretically, there should be no motion; therefore, any velocity value is model error. 

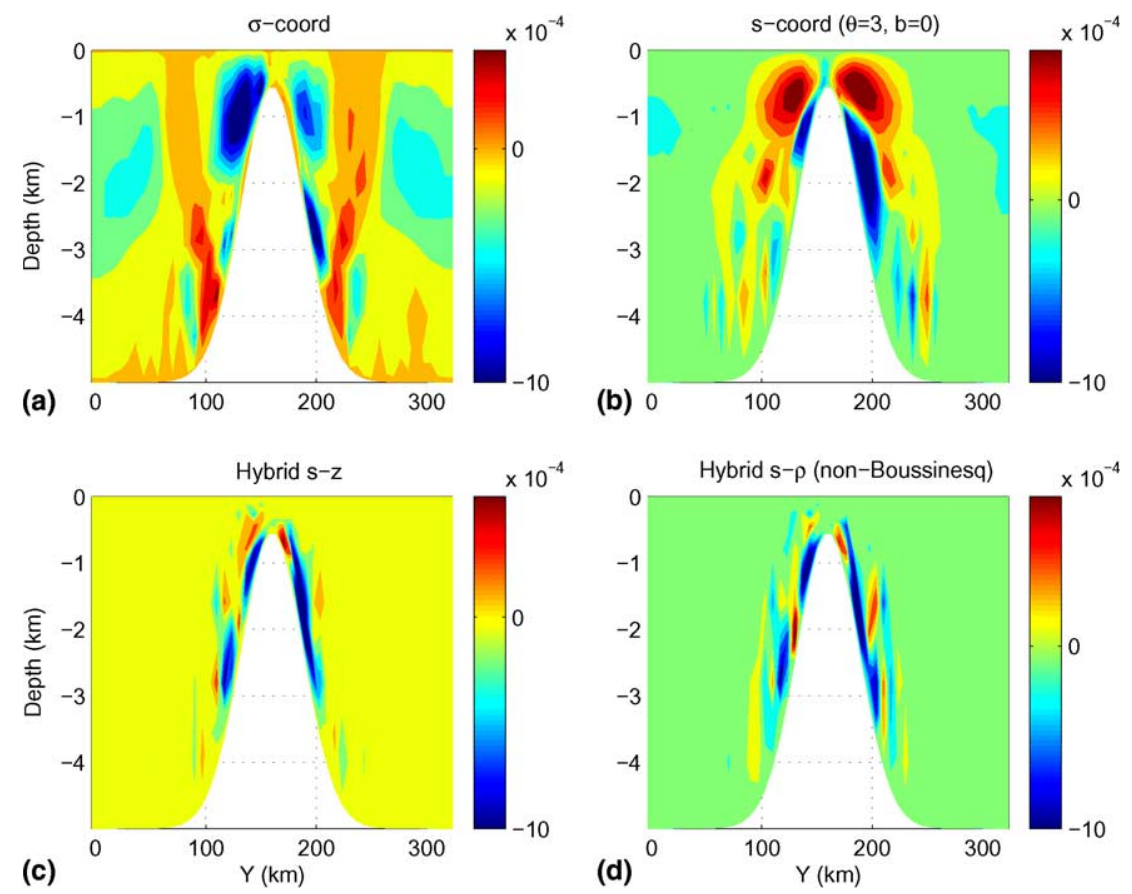

Fig. 4. Cross-section of vertical velocity $w$ at the end of 180 days. Notice that both $\sigma$-and $s$-coordinates (upper panels) generate errors in the whole domain while the two hybrid coordinates (lower panels) have the errors only near the sleep topography.

Fig. 4 gives the distributions of the erroneous vertical velocity after 180 days. It can be seen that both $\sigma$ - and $s$-experiments show errors in the whole domain, while the two hybrid coordinate cases have errors only near the bottom. It is well-known that the model errors mainly come from the pressure gradient formulations because the computational levels cannot be aligned with isopycnals near steep topography (Haney, 1991; Beckmann and Haidvogel, 1993). The problem becomes more severe if the computational levels also cross the isopycnals near the surface layer because the surface errors will be carried out to the deep ocean, as shown by the $\sigma$-experiment. The $s$-coordinates clearly improve the $\sigma$-coordinate errors near the surface, but not necessarily near the bottom, which depends on the choices of $\theta, b$ and the "optimal" weighting, as indicated by Shchepetkin and McWilliams (2003). The two hybrid cases further reduce the errors by aligning computational levels with density levels in the whole water column except for the bottom boundary layer.

To further understand the dynamic process of the model errors, we have also calculated the time series of mean kinetic energy (MKE) and maximum horizontal velocity $\left(V_{\max }\right)$ in Fig. 5. First, we have noticed that the traditional $\sigma$-coordinate gives the worst erroneous energy and horizontal maximum velocity, consistent with the above vertical velocity structures. Its mean kinetic energy is about $0.006 \mathrm{~m}^{2} \mathrm{~s}^{-2}$ and maximum velocity is about $0.55 \mathrm{~m} \mathrm{~s}^{-1}$ after 180 days. The $s$-coordinate is the second worst with the MKE error of $0.001 \mathrm{~m}^{2} \mathrm{~s}^{-2}$ and the $V_{\max }$ error of $0.35 \mathrm{~m} \mathrm{~s}^{-1}$, and the two hybrid cases are equally the best with MKE error of $0.00004 \mathrm{~m}^{2} \mathrm{~s}^{-2}$ and $V_{\max }$ error of $0.08 \mathrm{~m} \mathrm{~s}^{-1}$ after 180 days. A good feature of these experiments is that the errors 

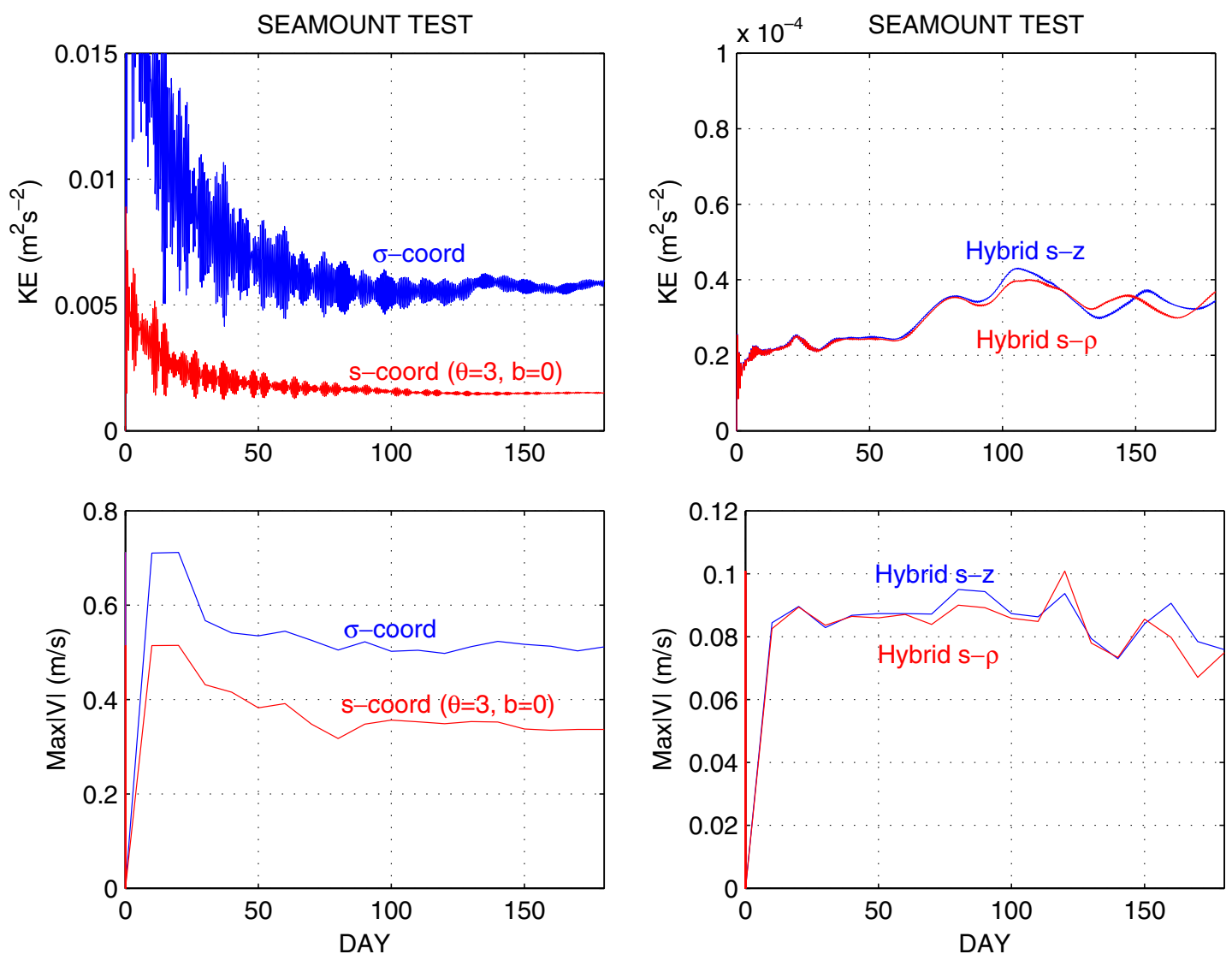

Fig. 5. Time series of mean kinetic energy (upper panels) and maximum horizontal velocity (lower panels) from the four experiments with different coordinate system. Notice the scale difference between the terrain-following coordinate models (left panels) and the two hybrid models (right panels). The hybrid coordinates reduce the MKE error by a factor of 10 and the $V_{\max }$ error by a factor of 5 , respectively.

do not increase after 150 days. Secondly, we compare these results with those (their Fig. 9) of Shchepetkin and McWilliams (2003). Their modified pressure gradient scheme has two steps: first fitting the density profile into polynomials and then applying the (standard or weighted) Jacobian schemes in the pseudo-flux form. It should be noticed that the polynomial fit approach might change the original data (e.g., the e-fold function cannot be fit exactly by a polynomial), and may be inconsistent with our tests here. Nevertheless, we noticed that our $\sigma$ - and $s$-experiments gave larger errors than those corresponding cases (POM and Lin97) in Shchepetkin and McWilliams (2003). However, our hybrid experiments achieve better results than their CubicA case and almost as good as their best $\mathrm{CubicH}$ case. The CubicA and $\mathrm{CubicH}$ give the MKE error of $0.00005 \mathrm{~m}^{2} \mathrm{~s}^{-2}$ and $0.00001 \mathrm{~m}^{2} \mathrm{~s}^{-2}$, and the $V_{\max }$ error of $0.08 \mathrm{~m} \mathrm{~s}^{-1}$ and $0.05 \mathrm{~m} \mathrm{~s}^{-1}$, respectively.

We have also repeated the same seamount test of Song and Wright (1998) by removing a background density in the initial fields. The maximum velocity error after 30 days is about $0.0375 \mathrm{~cm} \mathrm{~s}^{-1}$ for the $\sigma$-coordinates and $0.0095 \mathrm{~cm} \mathrm{~s}^{-1}$ for the hybrid $s-z$ coordinates, i.e., the hybrid coordinate model reduces the velocity error by a factor of 4 . These results add support to the 
contention that the hybrid coordinates can reduce the pressure gradient errors by following isopycnals in the interior region and enhancing resolutions in the surface and bottom layers.

\subsection{Non-Boussinesq pacific ocean with nested eastern tropics}

The purpose of this demonstration has twofold: (a) to test the model's capabilities of handling large-scale, non-Boussinesq circulation problems; and (b) to test the model's capabilities of handling multiscale problems by nesting a high-resolution coastal model into the basin-scale model. For this purpose, we have used the same model configuration for the two regions with different resolutions:

Non-Boussinesq Pacific Ocean: The basin-scale model has a horizontal resolution of a $0.5^{\circ}$ by $0.5^{\circ}$ grid from $45^{\circ} \mathrm{S}$ to $65^{\circ} \mathrm{N}$ and from $100^{\circ} \mathrm{E}$ to $280^{\circ} \mathrm{E}$. The model starts with initial conditions of annual mean temperature and salinity of Levitus et al. (1994).

Eastern Tropical Region: The regional model has a horizontal curvilinear grid following the coastline from $10^{\circ} \mathrm{S}$ to $20^{\circ} \mathrm{N}$ and from $210^{\circ} \mathrm{E}$ to $280^{\circ} \mathrm{E}$ with grid sizes from 5-km near the coast to $15-\mathrm{km}$ grid offshore. The model starts with initial conditions of the 10th-year output of the Pacific basin model.

Both models are forced by the monthly mean air-sea fluxes of momentum, heat, and fresh water derived from the Comprehensive Ocean-Atmosphere Data Set (COADS) climatology. The water depth is divided into 20 vertical levels for the basin and 16 levels for the eastern tropics model. Their bottom topographies are derived from the ETOP05 database and interpolated into the model grids. The newly introduced $s p$-coordinate system is used in both models.

Non-Boussinesq models have not been widely used to simulate regional- and basin-scale ocean circulations. To our knowledge, this is probably the first such kind model with both topographyfollowing and non-Boussinesq features for the Pacific basin with a nested regional model. Fig. 6 gives the nested-model configuration and the model sea-surface salinity after 11 years simulation. It is known that the eastern Pacific of the cold tongue region off the Central America is challenging for regional modeling because of its complex coastal geometry and intense internal and external interaction between local eddies and large-scale tropical instability waves. For demonstration purpose, only one-way nesting is applied, i.e., the open boundary conditions for the coastal model are extracted from the large-scale Pacific model, but no feedback information goes to the Pacific model. Nevertheless, the high-resolution regional model gives the active eddy fields in the eastern tropics, which cannot be resolved by the basin-scale Pacific model. The tropical instability waves and trains of large-amplitude eddies, propagating westward, with a wavelength of $1100 \mathrm{~km}$ and a phase speed of about $0.5 \mathrm{~m} \mathrm{~s}^{-1}$, are important features of the tropical oceans. Comparison with TOPEX/Poseidon data indicates that they could have strong bottom pressure signals, likely alias the GRACE measurements, which have a monthly-sampling period (Song and Zlotnicki, 2003).

As our models are non-Boussinesq, we have calculated the ocean bottom pressure (OBP) anomaly and its corresponding sea-surface height ( $\mathrm{SSH}$ ) anomaly in Fig. 7. The bottom pressure variable is directly calculated using conservation of mass, and the surface elevation is retrieved from the bottom pressure while considering the effect of heat expansion and contraction processes. The SSH anomaly (upper panel) represents the changes of surface elevation and the OBP anomaly 


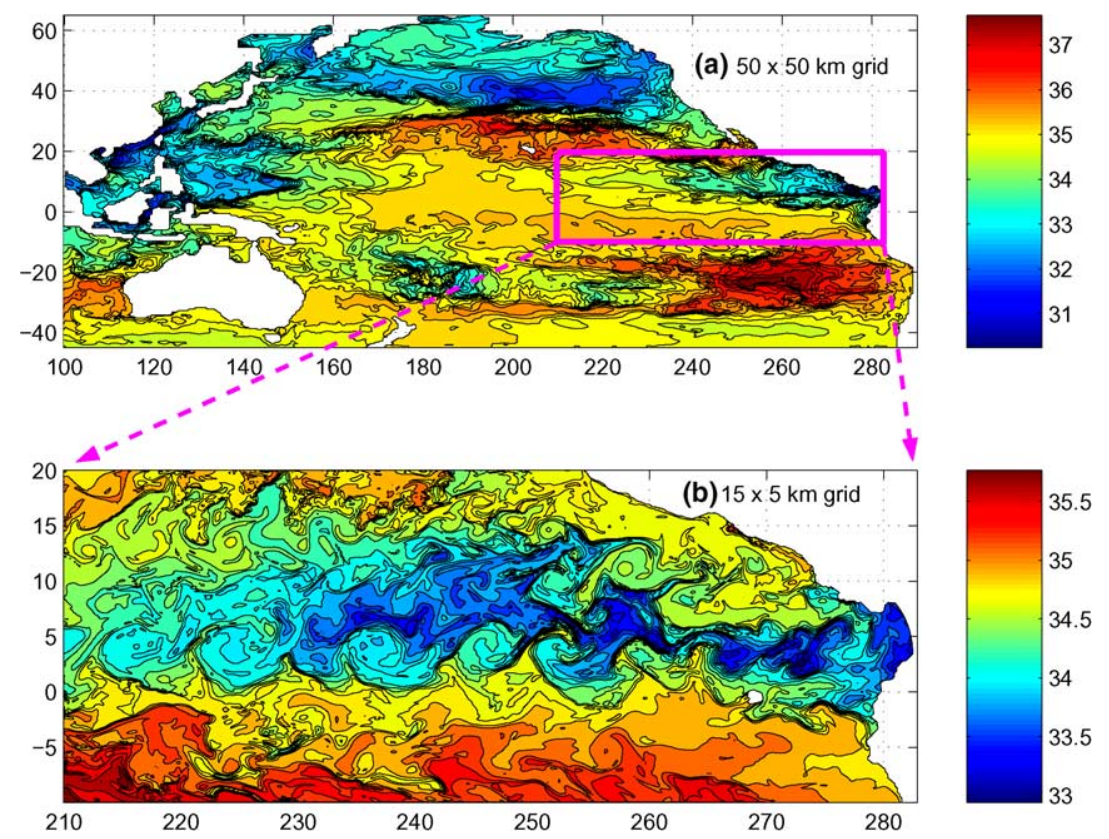

Fig. 6. Model configuration of non-Boussinesq Pacific Ocean with the nested eastern Tropics: a snapshot of sea-surface salinity after 11 years from the nested models. The box edges in the Pacific model are open boundary conditions $(u, v, T, S, \zeta, \bar{u}, \bar{v})$ for the nested eastern Tropics model.

(lower panel) represents the total mass of changes of the water column, both with the annual mean removed. The higher values in red and orange colors indicate gaining volume/mass, while the lower values in blue and green colors indicate losing water volume/mass, in comparison with their mean state. Clearly, the ocean's volume change does not necessarily mean the mass changes. We also give the surface velocity and temperature in Fig. 8. These results are reasonably good by comparison with known circulation features. For example, the Kuroshio separation is at the right location, even with such a coarse grid, and the large-scale features of the equatorial cold-tongue is well resolved, but not the filament features associated with the equatorial instability eddies, which are revealed clearly in the finer-grid model.

To focus on the eastern tropical region, we give the corresponding surface velocity and temperature fields for the tested model in Fig. 9. Notice that the train of eddies centered at $4^{\circ} \mathrm{N}$ and the cold tongue with the cusp-shaped filaments are known features from observations (Song and Zlotnicki, 2003), which could not be resolved in the coarser-grid Pacific model. It clearly shows that the consistent coupling between the two models allows the tropical instability eddies to propagate out of the domain without artificial reflection near the open boundaries.

It should be pointed out that one of the most challenging tasks in coastal ocean modeling is to obtain proper open boundary conditions. To have the highest possible resolution for a coastal region, the model domain is often limited and cut off from large-scale ocean circulation systems. In reality, a coastal region is not the only source of the local phenomena: it is forced by remotely large-scale dynamics and needs outside information to get the local solution useful. The performance of the coastal model greatly depends on the consistency between the external information 


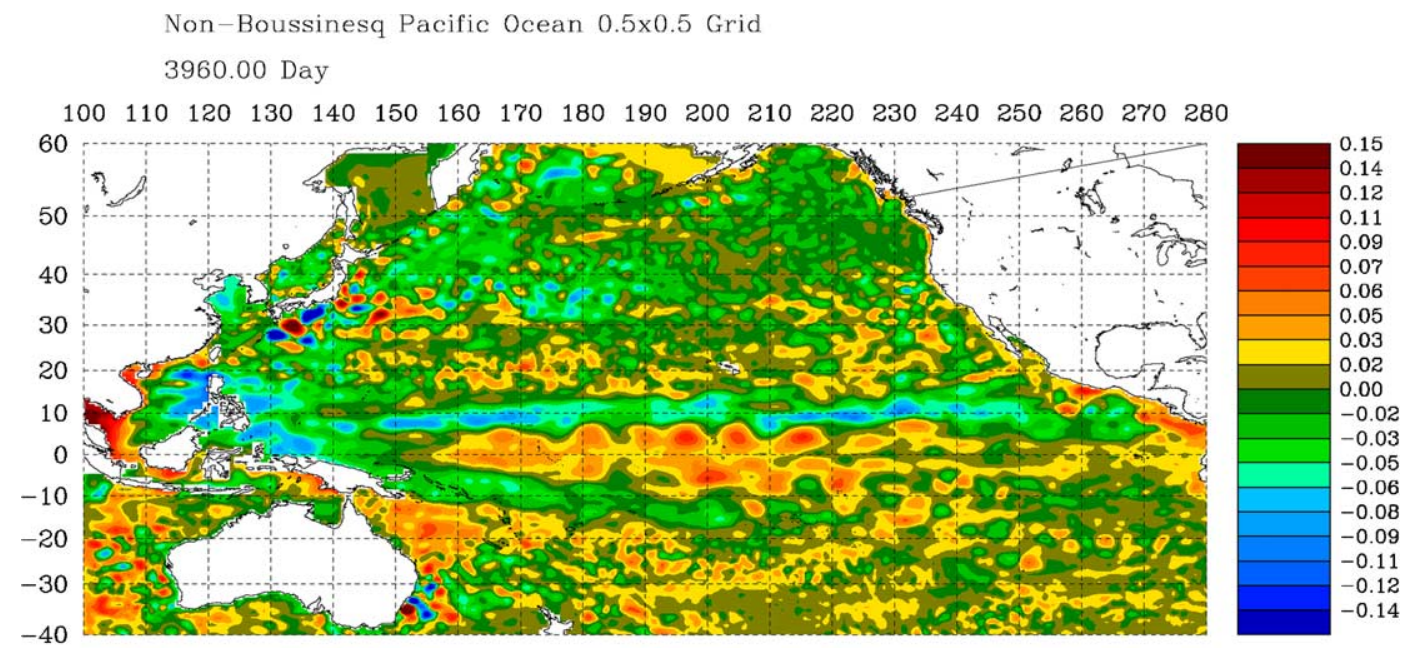

(a)

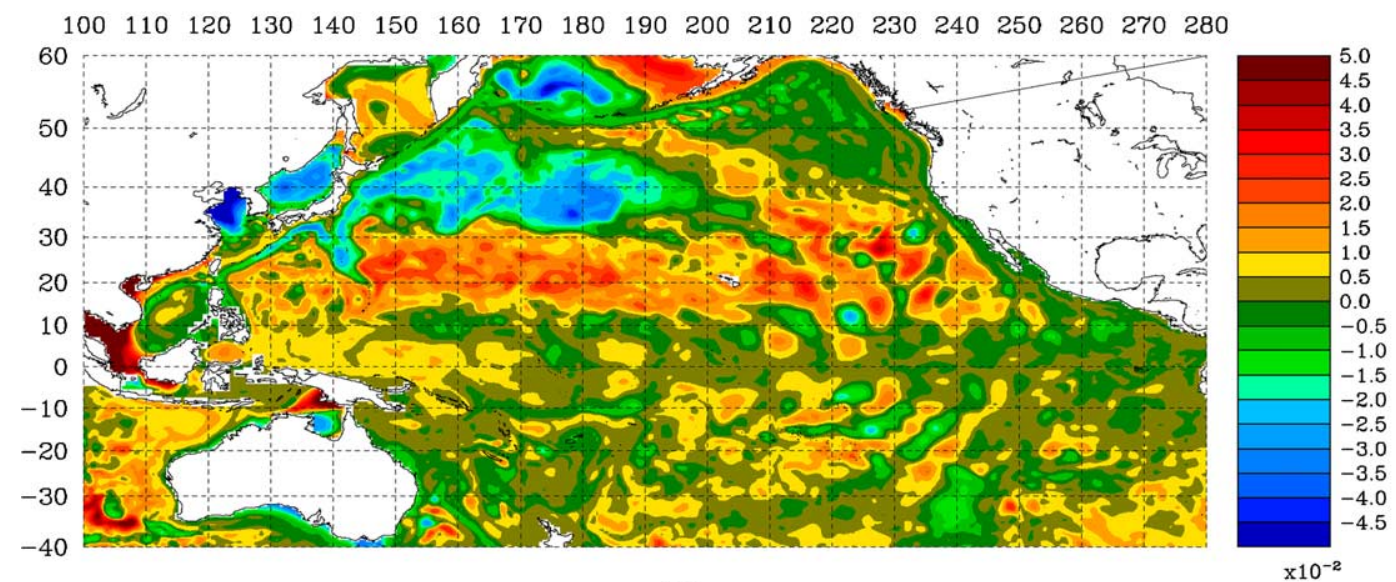

(b)

Fig. 7. A snapshot of non-Boussinesq model results after 11 years (or 3960 days): (a) sea-surface height, representing volume change; (b) bottom pressure anomaly, representing mass change. Note the bottom pressure anomaly is normalized by $\left(P_{\mathrm{b}}-P_{\mathrm{b}}^{0}\right) / g \rho_{0}$, where $P_{\mathrm{b}}$ is the bottom pressure and $P_{\mathrm{b}}^{0}$ is the time mean pressure. Units are meters for $\mathrm{SSH}$ and $10^{-2} \mathrm{~m}$ (mbar) for $P_{\mathrm{b}}$.

and the boundary algorithms. Therefore, the use of the multiscale nested models is a compromise for obtaining high-resolution in the sub-domain of interest and minimizing the overall computational cost, without losing the influence of the large-scale dynamics. Here we demonstrate that such a multiscale nested task can be carried out easily within the same model for both large-scale information and local fine-scale dynamics. Though the nesting task can be carried out with different single-coordinate models, modelers have to run different numerical models in order to use various coordinate features for the two regions with different scales. It is often difficult to maintain multiple codes, perhaps even more difficult is to make them physically and numerically compatible with each other. The present modeling approach clearly provides a better way of dealing such 
Non-Boussinesq Pacific Ocean $0.5 \times 0.5$ Grid

3960.00 Day

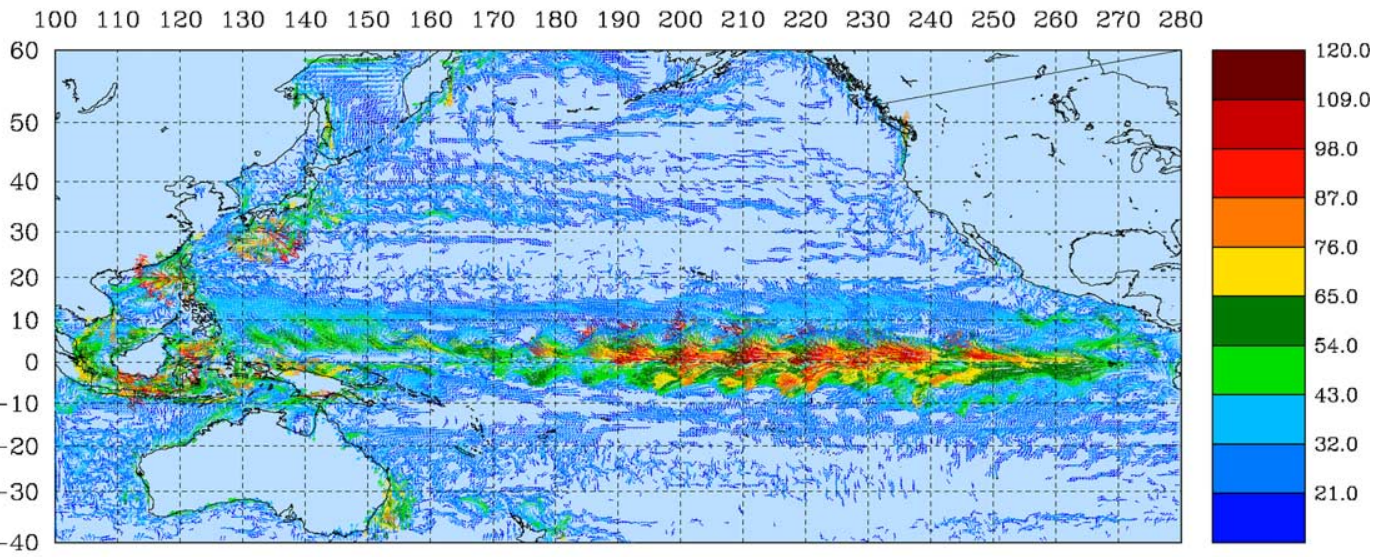

(a) Velocity Vectors $(\mathrm{cm} / \mathrm{s})$ at Level 20

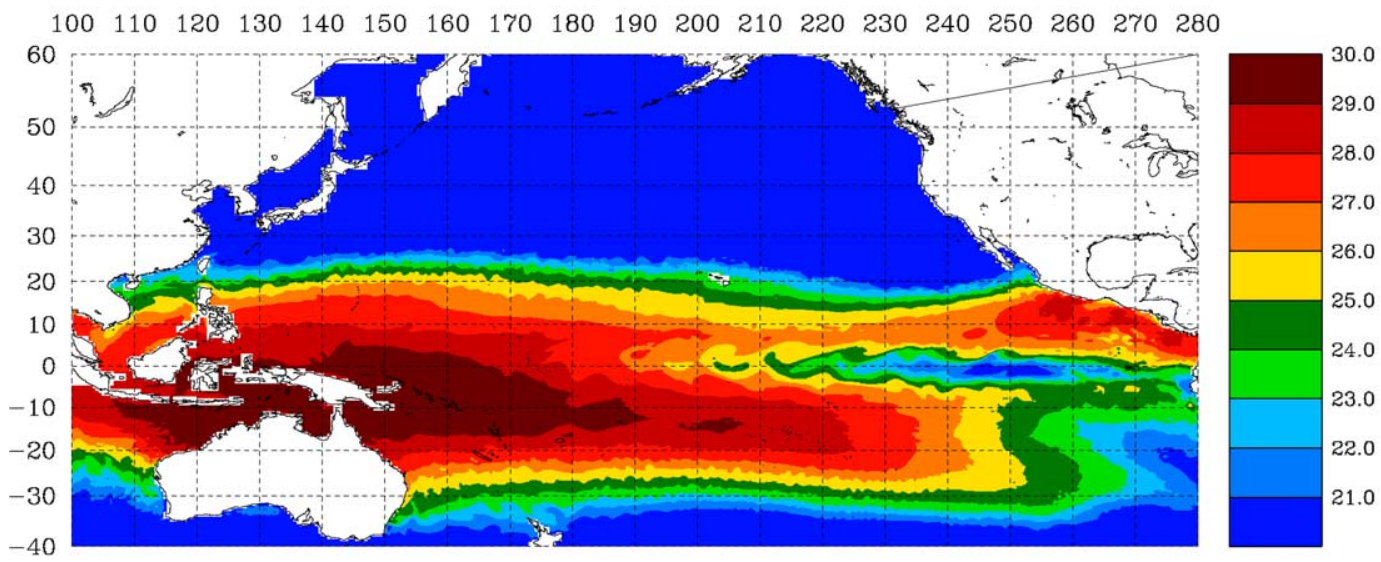

(b) Potential Temperature (C) at Level 20

Fig. 8. A snapshot of the non-Boussinesq Pacific model after 11 years: (a) sea-surface velocity, color bar represents the speed; (b) sea-surface temperature $\left({ }^{\circ} \mathrm{C}\right)$, showing the well-developed equatorial cold tongue in the eastern tropical region.

kinds of problems by using various vertical coordinate systems on the same numerical platform. The inclusion of the non-Boussinesq physics in the model does not incur computational expense with our parametric coordinate formulation. Such extended model capabilities are needed in currently used community ocean models to faithfully represent satellite sensing data (Huang et al., 2001).

\subsection{Global ocean circulation in S-coordinates}

The purpose of this test is to demonstrate the capability of the new model in handling globalscale applications for long-term simulations. The global model covers the world ocean from $75^{\circ} \mathrm{S}$ 


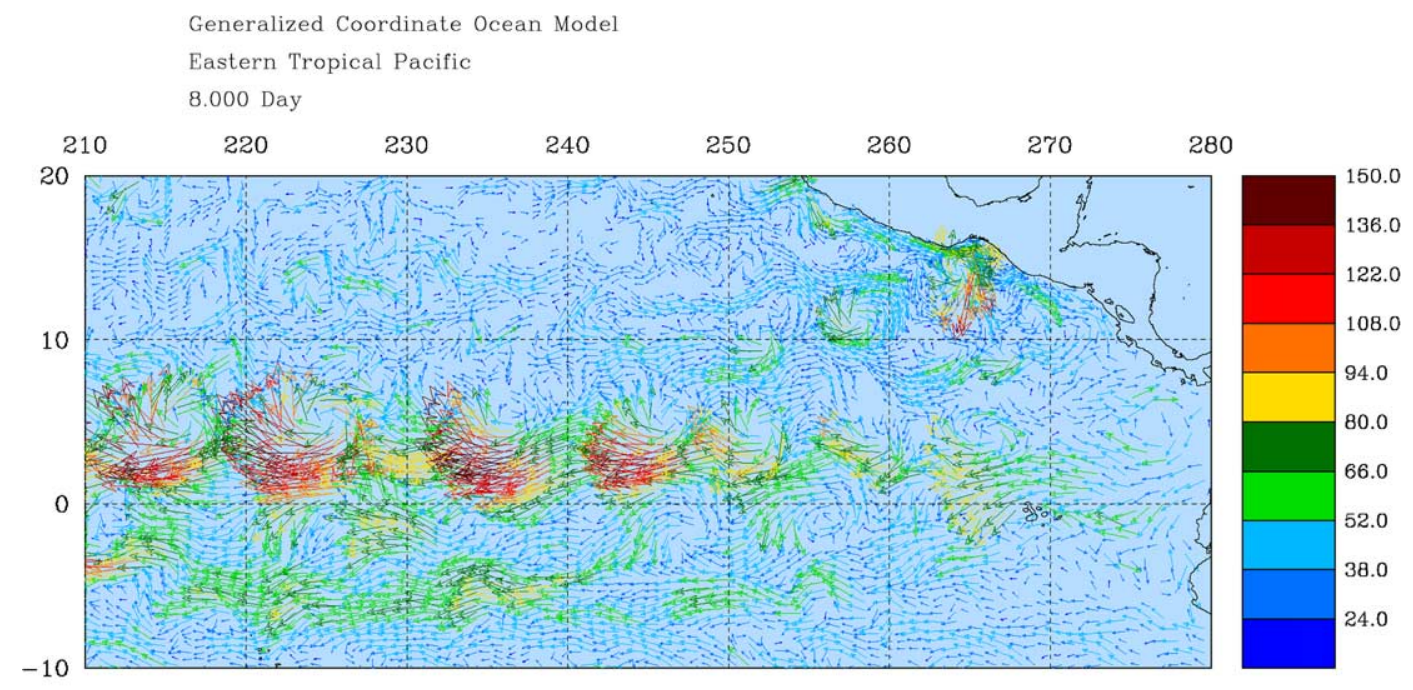

(a) Total Velocity Vectors $(\mathrm{cm} / \mathrm{s})$ at Level 16

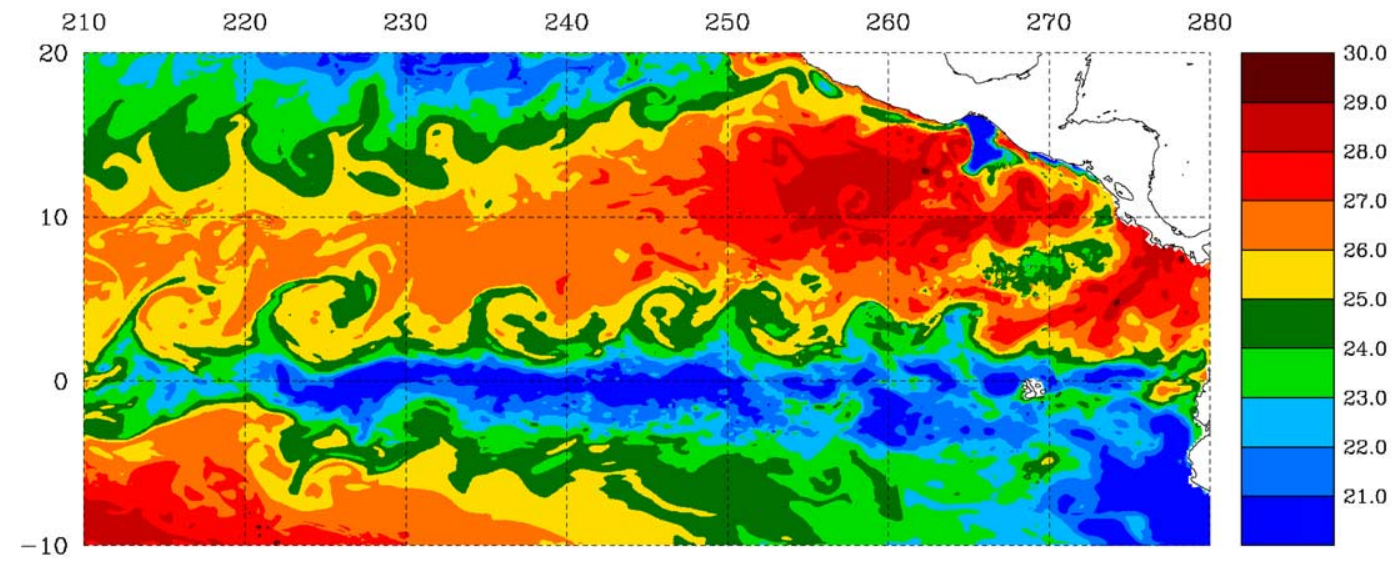

(b) Potential Temperature (C) at Level 16

Fig. 9. Same as the Fig. 8, but for the nested eastern Tropics model after one year simulation: (a) sea-surface velocity, color bar represents the speed; (b) sea-surface temperature $\left({ }^{\circ} \mathrm{C}\right)$. Please notice the train of tropical instability eddies (upper panel) and the cusp of filaments generated by the eddies. These detail features are not seen in the coarser-grid Pacific model.

to $75^{\circ} \mathrm{N}$ with realistic geometry. The model grid resolution is $0.5^{\circ} \times 0.5^{\circ}$, with enhanced resolution to $1 / 3^{\circ}$ in the range of $30^{\circ} \mathrm{S}$ to $30^{\circ} \mathrm{N}$. The bottom topography is derived from the ETOPO5 database and is interpolated into the model grid. Initial $T$ and $S$ are obtained from the Levitus et al. (1994) mean-monthly climatology data. Surface boundary conditions include heat and fresh water fluxes and wind stress obtained from NCEP/NCAR reanalysis data. There are 20 vertical levels with 10 levels in the upper 500-meter depth and the other 10 levels in the lower layer, using the $s$-coordinate system of Song and Haidvogel (1994) with $\theta=5$ and $b=0$. Although this 
generalized terrain-following model (SCRUM/ROMS) has been widely used for coastal and regional oceans, it has not been well tested for global-scale problems.

The model is first spun-up for 50 years with annual-mean NCEP/NCAR forcing. Time series of global averaged kinetic energy and potential energy are shown in Fig. 10 to demonstrate the extent to which a quasi-steady state has been reached at the end of the 50-year spun-up run. The early 10 years of the run are dominated by a kind of geostrophic adjustment process in which the potential energy convert rapidly to the kinetic energy, which is then subject to dissipation. The values of horizontal viscosity and diffusivity used in the model are 400 and $100 \mathrm{~m}^{2} \mathrm{~s}^{-1}$, respectively. The physical reason for the energy conversion has been discussed by Song and Wright (1998) as the advective elimination of the inconsistency between the finite-difference nature of the model and the initial state. Such adjustment process is required for energetic consistency and the success of long-term integrations. However, the dissipation process is extremely slow as it can be seen that the potential energy still increases slightly after 50 years, but has reached a quasi-steady state after 30 years.
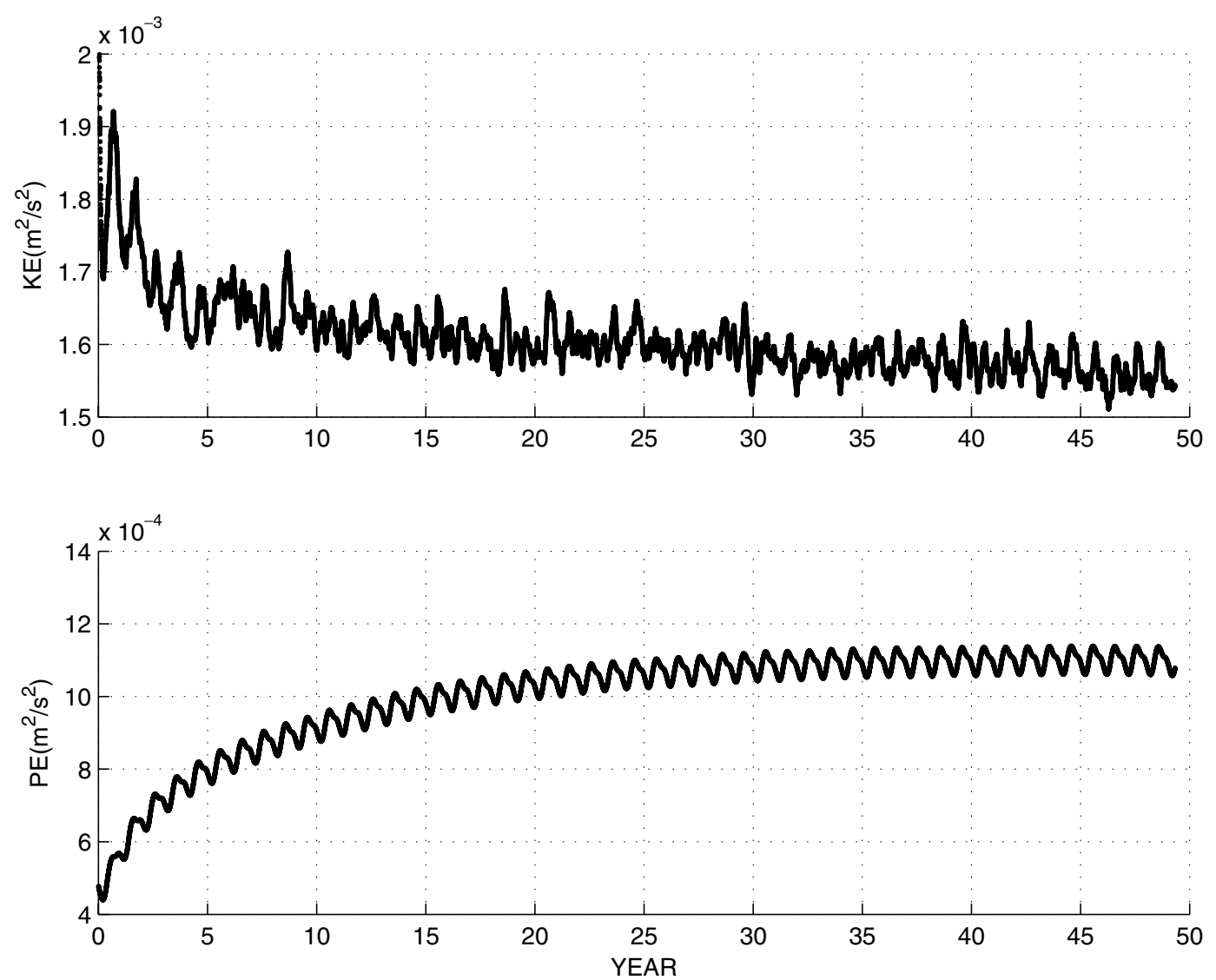

Fig. 10. Upper panel is the globally averaged kinetic energy and the lower panel is the averaged potential energy during the 50-year spun-up run from the $s$-coordinate global model. 
As we have emphasized the topographic effect on ocean circulations, we show the zonal section at $60^{\circ} \mathrm{S}$ along the Antarctic Circumpolar Current (ACC) in Fig. 11 for the temperature (upper panel) and salinity (lower panel) after the 50-year simulation. The four ridges are the major topographic features in the Southern Ocean. They indeed have a significant effect on the temperature and salinity structures; particularly, the temperature structure is clearly divided by the ridges into four groups.

In Fig. 12, we give the sea-surface elevation and the velocity fields. These results are reasonably good in comparison with known observations, such as the separation latitudes of the Kuroshio and the Gulf Stream, the North Atlantic current system, the Agulhas Retroflection, the equatorial current system, and the strength of the ACC. Some deficiencies can also be seen. For example, there is no noticeable signature of the Azores current at about $35^{\circ} \mathrm{N}$ in the Atlantic Ocean, which may be due to the insufficient resolution of the model. We will continue the spun-run of the model
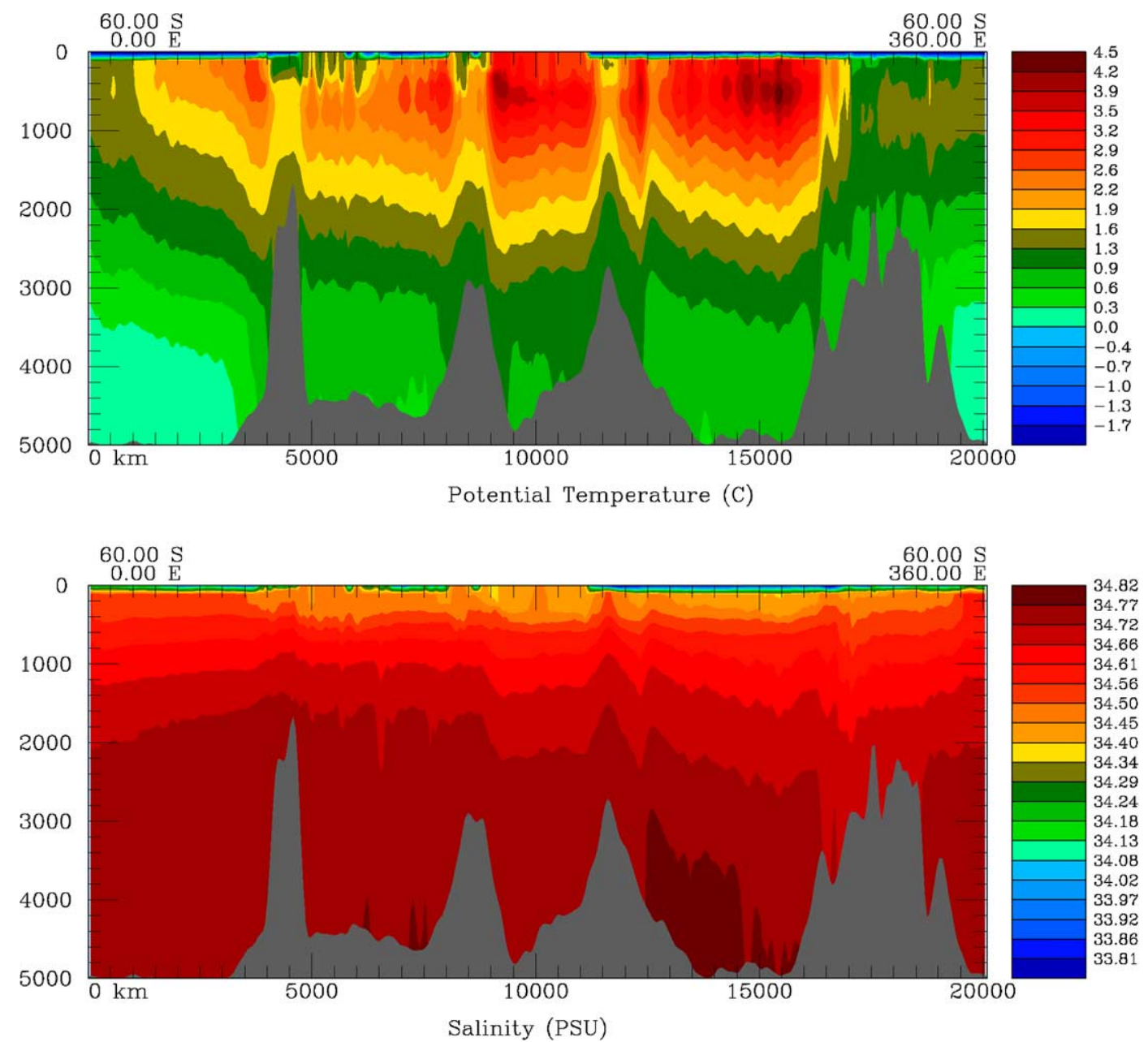

Fig. 11. Zonal section at $60^{\circ} \mathrm{S}$ along the ACC: (Upper panel) vertical temperature structure $\left({ }^{\circ} \mathrm{C}\right.$ ) and (Lower) vertical salinity structure (psu). Both are monthly averaged at day 17,505 from the $s$-coordinate global model. 

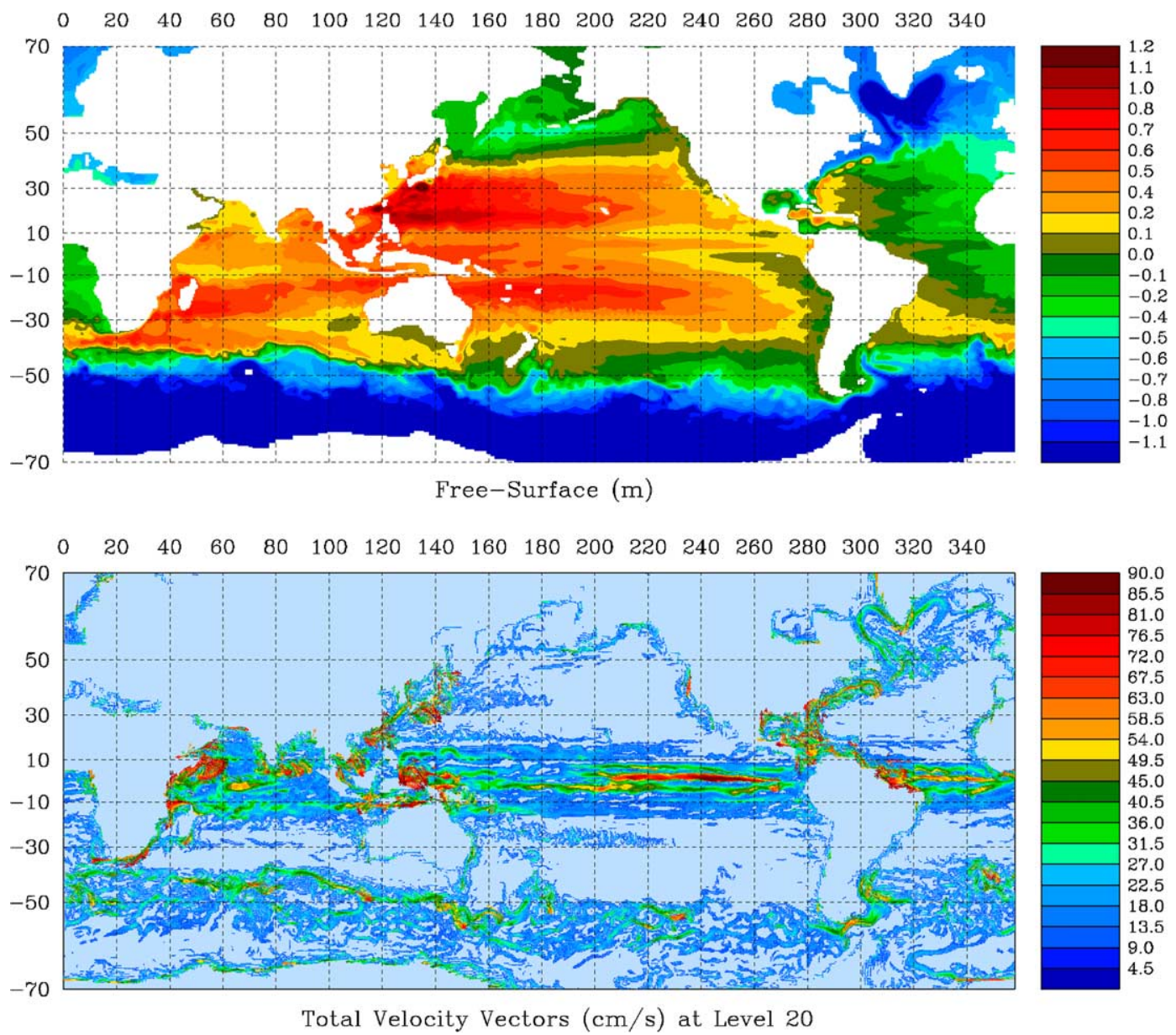

Fig. 12. Global ocean simulation after 50 years: (upper) sea-surface elevation and (lower) velocity fields. Both are monthly averaged at day 17,505 . Color bars are meter and $\left(\mathrm{cm} \mathrm{s}^{-1}\right)$, respectively.

to a steady state, then apply the realistic atmospheric forcing for quantitative comparisons with observations. Nevertheless, the initial 50-year simulations illustrate that the model with the new scheme is stable for long-term integrations and capable of resolving global-scale problems with topography-following coordinate system.

\section{Discussions and summary}

Traditional ocean models have been built upon an "explicit coordinate" formulation; the model numerical design is based on a single (fixed) vertical coordinate system in the basic ocean equations. Therefore it results in a variety of model classes with different numerical configurations as mentioned in the introduction. Users have limited freedom to choose different coordinate systems in a given model configuration. On the contrary, the present model is developed upon an 
"parametric coordinate" formulation; the model numerical design is based on an arbitrary (parametric) vertical coordinate system. Users have the freedom to choose combined coordinate features by selecting input parameters without altering the numerical configuration. Therefore, it offers an easy way to take advantage of various coordinate features for multiscale modeling studies. The new model extends the previous $s$-coordinate model (Song and Haidvogel, 1994) in two aspects:

(1) Numerically it offers flexible choices of the optimal or hybrid features of many traditional coordinate systems, including the original $s$-coordinate system;

(2) Physically it allows both Boussinesq and non-Boussinesq flow conditions within the same numerical configuration.

Adding these two extensions does not necessarily incur computational expense. Combining these two extensions into a single model allows users to take advantages of various coordinate combinations for multiscale applications. The inclusion of mass-conserving physics in the model allows users to explore both Boussinesq and non-Boussinesq conditions in the same model configuration. Although the effects of non-Boussinesq approximations on the model simulation of the ocean circulation remain unclear (Mellor and Ezer, 1995; Huang et al., 2001; Greatbatch et al., 2001; Losch et al., 2004), here we provide an alternative to relax the Boussinesq approximations in the terrain-following coordinate system (Song and Zlotnicki, 2003). This is particularly useful in nested modeling, in which the large-scale model that could be global needs characteristics of $z$-coordinates or non-Boussinesq approximations for a proper representation of thermodynamical and biogeochemical processes; while the nested small-scale model that could be coastal or regional needs to resolve boundary layer dynamics and fine-scale topographic features.

So far, we have demonstrated that the parametric modeling techniques can be easily implemented into an existing ocean model, like SCRUM/ROMS. We have also shown that the model is capable of resolving multiscale ocean-related problems from coastal to basin-scale and to global ocean within a single numerical configuration. These test problems are chosen to have different scales and challenging topographic features. In the coastal canyon problem, we have demonstrated that the predominant $z$-coordinate option of the model is able to perform as well as $\sigma$-coordinate models. In the regional model with a seamount topography, we have found that the hybrid $s-z$ and $s-\rho$ coordinates can be used to reduce pressure gradient errors greatly. In the basin-scale Pacific model, we are able to simulate the large-scale circulations with both non-Boussinesq and topography-following features and a nested regional model with active tropical instability waves. In the global ocean model, we have demonstrated that the $s$-coordinate system, widely used in coastal and regional ocean modeling, is capable of handling the global-scale problems for long-term simulations.

Clearly, this paper could not possibly test and include all the available options of the proposed modeling approach. Here we have only reported some of the commonly used hybrid configurations with the topography-following feature for coastal oceans. Some new features should be explored by expert users, who often have the best knowledge to choose the optimal coordinate structure for their specific applications. Many of the advanced features of the model are still under testing and will be reported in following papers. Obvious future work includes comparisons with truly $z$-level and isopycnal models. These would need to run different models or significantly 
modify the code to allow step-like/shaved-cell capabilities (Adcroft et al., 1997) and truly isopycnals (Bleck, 2002), which are beyond the scope of this paper.

Finally, it is interesting to note that most of the traditional coordinate systems in ocean modeling, such as the $z, \sigma$, and $p$-coordinates, are directly borrowed from atmospheric modeling (Kasahara, 1974). However, the ocean and the atmosphere have fundamental differences. One of these is that the ocean has both surface and bottom boundary layers that interact with atmosphere and bottom topography simultaneously. The two boundary layers meet in the coastal oceans, creating the most dynamically and biologically active regions of the ocean as well as great challenges for ocean model design. Mainly due to this fundamental difference, we have paid special attention to the two boundary layers in the model design, as schematically shown in Fig. 1, and introduced innovative numerics particularly suitable for ocean-related problems. These innovative techniques, such as the stretched pressure $s p$-coordinates, the Jacobian formulation of the pressure gradients, and the newly introduced parametric function, $\phi=H_{z} B_{z}$, have only been tested in ocean modeling. Whether they can be used for atmospheric modeling has not been verified.

\section{Acknowledgments}

The research described here was conducted at the Jet Propulsion Laboratory, California Institute of Technology, under contract with the National Aeronautics and Space Administration, funded by the Office Naval Research (N00014-03-IP-20059) and the 2002 Caltech President's Fund Award.

\section{References}

Adcroft, A., Hill, C., Marshall, J., 1997. Representation of topography by shaved cells in a height coordinate ocean model. Mon. Weather Rev. 125, 2293-2315.

Arakawa, A., Lamb, V.R., 1977. Computational design of the basic dynamic processes of UCLA general circulation model. Methods in Comput. Phys., vol. 17. Academic Press, New York/London, pp. 173-265.

Arakawa, A., Suarez, M.J., 1983. Vertical differencing of the primitive equations in Sigma Coordinates. Mon. Weather Rev. 111, 34-45.

Baer, F., Ji, M., 1989. Optimal vertical discretization for atmospheric models. Mon. Weather Rev. 117, $391-406$.

Beckmann, A., Döscher, R., 1997. A method for improved representation of dense water spreading over topography in geopotential-coordinate models. J. Phys. Oceanogr. 27, 581-591.

Beckmann, A., Haidvogel, D.B., 1993. Numerical simulation of flow around an isolated seamount. Part I: Problem formulation and model accuracy. J. Phys. Oceanogr. 23, 1736-1753.

Bell, J.M., 1999. Vortex stretching and bottom torques in the Bryan-Cox ocean circulation model. J. Geophys. Res. 104, 23, 545-23, 563.

Bleck, R., 1978. Finite difference equations in generalized vertical coordinates. Part I: Total energy conservation. Contrib. Atmos. Phys. 51, 360-372.

Bleck, R., 2002. An ocean general circulation model framed in hybrid isopycnic-Cartesian coordinates. Ocean Model. 37, 55-88.

Bleck, R., Benjamin, S., 1993. Regional weather prediction with a model combining terrain-following and isentropic coordinates. Part I. Mon. Weather Rev. 121, 1770-1785. 
Bleck, R., Boudra, D.B., 1981. Initial testing of a numerical ocean model using a hybrid (quasi-isopycnic) vertical coordinate. J. Phys. Oceanogr. 11, 755-770.

Bleck, R., Rooth, C., Hu, D., Smith, L., 1992. Salinity-driven thermocline transients in a wind- and thermohalineforced isopycnic coordinate model of the North Atlantic. J. Phys. Oceanogr. 22, 1486-1505.

Blumberg, A.F., Mellor, G.L., 1987. A description of a three-dimensional coastal ocean circulation model. In: Heaps, N. (Ed.), Three-Dimensional Coastal Ocean Models, vol. 4, AGU, 208 pp.

Boussinesq, J., 1903. Theorie Analyque De La Chaleur, vol. 2. Gauthier-Villars, Paris.

Bryan, K., 1969. A numerical method for the study of the circulation of the World Ocean. J. Comp. Phys. 4, 347-376.

Chassignet, E.P. et al., 2000. DAMEE-NAB: the base experiments. Dynam. Atmos. Oceans 32, 155-183.

Chu, P.C., Fan, C., 1997. Sixth-order difference scheme for sigma coordinate ocean models. J. Phys. Oceanogr. 27, 2064-2071.

Cox, M.D., 1984. A primitive equation three-dimensional model of the ocean. GFDL Ocean Group Tech. Rep. 1. Princeton University, $250 \mathrm{pp}$.

Deleersnijder, E., Seekers, J., 1992. On the use of the $\sigma$-coordinate system in regions of large bathymetric variations. J. Marine Syst. 3, 381-390.

De Szoeke, R.A., Samelson, R., 2002. The duality between the Boussinesq and non-Boussinesq hydrostatic equations of motion. J. Phys. Oceanogr. 32, 2194-2203.

Drijfhout, S., Heinze, C., Latif, M., Maier-Reimer, E., 1996. Mean circulation and internal variability in an ocean primitive equation model. J. Phys. Oceanogr. 26, 559-580.

DYNAMO group, 1997. DYNAMO - Dynamics of North Atlantic Models: Simulation and assimilation with higherresolution models. Ber. Inst. f. Meereskunde Kiel, 294, 333 pp.

Ezer, T., Arango, H., Shchepetkin, A.F., 2002. Developments in terrain-following ocean models: intercomparisons of numerical aspects. Ocean Model. 4, 249-267.

Fletcher, C.A.J., 1991. Computational Techniques for Fluid Dynamics 1: Fundamental and General Techniques. Springer-Verlag, Berlin, Heidelberg, $401 \mathrm{pp}$.

Fu, L.-L., 1983. Recent progress in the application of satellite altimetry to observing the mesoscale variability and general circulation of the oceans. Rev. Geophys. Space Phys. 21, 1657-1666.

Gerdes, R., 1993. A primitive equation ocean circulation model using a general vertical coordinate transformation, I. Description and testing of the model. J. Geophys. Res. 98 (C8), 14683-14701.

Griffles, S.M., Böning, C., Bryan, F.O., Chassignet, E.P., Gerdes, R., Hasumi, H., Hirst, A., Treguier, A.M., 2000. Developments in ocean climate modelling. Ocean Model. 2, 123-192.

Greatbatch, R.J., Lu, Y., Cai, Y., 2001. Relaxing the Boussinesq approximation in ocean circulation models. J. Atmos. Oceanic Tech. 18, 1911-1923.

Haidvogel, D.B., Beckmann, A., 1998. Numerical models of the coastal ocean. In: Brink, K.H., Robinson, A.R. (Eds.), The Sea, vol. 10, pp. 457-482.

Haidvogel, D.B., Beckmann, A., 1999. Numerical Ocean Circulation Modeling. Imperial College Press, London, 318 pp.

Haidvogel, D.B., Arango, A., Hedstrom, K., Beckmann, A., Rizzoli, P., Shchepetkin, S., 2000. Model evaluation experiments in the North Atlantic basin: simulations in non-linear terrain-following coordinates. Dynam. Atmos. Oceans 32, 239-281.

Hallberg, R., 2000. Time integration of diapycnal diffusion and Richardson number-dependent mixing in isopycnal coordinate ocean models. Mon. Weather Rev. 128, 1402-1419.

Haney, R.L., 1991. On the pressure gradient force over steep topography in sigma coordinate ocean models. J. Phys. Oceanogr. 21, 610-619.

Hermeline, F., 2000. A finite volume method for the approximation of diffusion operators on distorted meshes. J. Comp. Phys. 160, 481-499.

Huang, R.X., Jin, X., Zhang, X., 2001. An ocean general circulation model in pressure coordinates. Adv. Atmos. Sci. $18,1-22$.

Hughes, C.W., Wunsch, C., Zlotnicki, V., 2000. Satellite peers through the oceans from space. EOS, Trans. Amer. Geophys. Union 81 (7), 68. 
Hughes, C.W., De Cuevas, B.A., 2001. Why western boundary currents in realistic oceans are inviscid: A link between form stress and bottom pressure torques. J. Phys. Oceanogr. 31, 2871-2885.

Iskandarani, M., Haidvogel, D.B., Boyd, J.P., 1995. A staggered spectral element model with applications to the oceanic shallow water equations. Int. J. Numer. Meth. Fluids 20, 393-414.

Kasahara, A., 1974. Various vertical coordinate systems used for numerical weather prediction. Mon. Weather Rev. 102, 509-522.

Killworth, P.D., Edwards, N.R., 1999. A turbulent bottom boundary layer code for using numerical ocean models. J. Phys. Oceanogr. 29, 1221-1238.

Konor, C.S., Arakawa, A., 1997. Design of an atmospheric model based on a generalized vertical coordinate. Mon. Weather Rev. 125, 1649-1673.

Lardner, R.W., Song, Y.T., 1991. An algorithm for three-dimensional convection and diffusion with very different horizontal and vertical length scales. Int. J. Numer. Meth. Eng. 32, 1303-1319.

Large, W.G., McWilliams, J.C., Doney, S.C., 1994. Oceanic vertical mixing: a review and a model with a nonlocal boundary layer parameterization. Rev. Geophys. 32, 363-403.

Levitus, S., Burgett, R., Boyer, T., 1994. World Ocean Atlas: Salinity and Temperature 3-4. NOAA Atlas NESDID, US Dept. Commerce.

Losch, M., Adcroft, A., Campin, J.M., 2004. How sensitive are coarse general circulation models to fundamental approximations in the equations of motion? J. Phys. Oceanogr. 34, 306-319.

Marshall, J., Adcroft, A., Hill, C., Perelman, L., Heisey, C., 1997. A finite-volume, incompressible Navier Stokes model for studies of the ocean on parallel computers. J. Geophys. Res. 102 (C3), 5753-5766.

Mellor, G.L., Ezer, T., 1995. Sea level variations induced by heating and cooling: an evaluation of the Boussinesq approximation in ocean models. J. Geophys. Res. 100 (C10), 20, 565-20, 577.

Mellor, G.L., Hakkinen, S., Ezer, T., Patchen, R., 2001. A generalization of a sigma coordinate ocean model and intercomparison of model vertical grids. In: Pinardi, N. (Ed.), Ocean Forecasting: Theory and Practice. SpringerVerlag, pp. 55-72.

Oey, L-Y., Mellor, G.L., Hires, R.I., 1985. A three-dimensional simulation of the Hudson-Raritan estuary. Part I: Description of the model and model simulations. J. Phys. Oceanogr. 15, 1676-1692.

Pacanowski, R., Ghanadesikan, A., 1998. Transient response in a $z$-level ocean model that resolves topography with partial cells. Mon. Weather Rev. 126, 3248-3270.

Phillips, N.A., 1957. A coordinate system having some spatial advantages for numerical forecasting. J. Meteor. 14, 184 186.

Pietrzak, J., Jakobson, J.B., Burchard, B., Vested, H.J., Petersen, O., 2002. A three-dimensional hydrostatic model for coastal and ocean modeling using a generalised topography following coordinate system. Ocean Model. 4, 173-205.

Roberts, M.J., Wood, R.A., 1997. Topographic sensitivity studies with a Bryan-Cox-type ocean model. J. Phys. Oceanogr. 27, 823-836.

Schopf, P.S., Loughe, A., 1995. A reduced gravity isopycnal ocean model: hindcasts of El Nino. Mon. Weather Rev. 123, 2839-2863.

Shchepetkin, A.F., McWilliams, J.C., 2003. A method for computing horizontal pressure-gradient force in an oceanic model with a nonaligned vertical coordinate. J. Geophys. Res. 108.

Shchepetkin, A.F., McWilliams, J.C., in press. The regional ocean modeling system: a split-explicit free-surface, topography-following-coordinate ocean model. Ocean Model.

Sheng, J., Wright, D.G., Greatbatch, R.J., Deitrich, D.E., 1998. CANDIE: a new version of the DeiCAST ocean circulation model. J. Atmos. Oceanic Tech. 15, 1414-1432.

Simmons, A.J., Burridge, M., 1981. An energy and angular-momentum conserving vertical finite-difference scheme and hybrid vertical coordinates. Mon. Weather Rev. 109, 758-766.

Smith, R.D., Dukowicz, J.K., Malone, R.C., 1992. Parallel ocean general circulation modeling. Physica D 60, $38-61$.

Song, Y.T., 1998. A general pressure gradient formation for ocean models. Part I: Scheme design and diagnostic analysis. Mon. Weather Rev. 126 (12), 3213-3230.

Song, Y.T., Chao, Y., 2000. An embedded bottom boundary layer formulation for $z$-coordinate ocean models. J. Atmos. Oceanic Tech. 7, 546-560. 
Song, Y.T., Haidvogel, D.B., 1994. A semi-implicit primitive equation ocean circulation model using a generalized topography-following coordinate system. J. Comp. Phys. 115, 228-244.

Song, Y.T., Wright, D., 1998. A general pressure gradient formation for ocean models. Part II: Energy, momentum, and bottom torque consistency. Mon. Weather Rev. 126 (12), 3231-3247.

Song, Y.T., Zlotnicki, V., 2003. Ocean bottom pressure waves detected in tropical Pacific. Geophys. Res. Lett. 31 (5), L05306.

Tapley, B.D., Betadpur, S., Watkins, M., Reigber, C., 2004. The gravity recovery and climate experiment: mission overview and early results. Geophys. Res. Lett. 31 (5), L09607.

Walsh, J.J., 1991. Importance of continental margins in the marine biogeochemical cycling of carbon and nitrogen. Nature 350, 53-55.

Zhu, Z., Thuburn, J., Hoskins, B.J., Haynes P.H., 1992. A vertical finite-difference scheme based on a hybrid $\sigma-\theta-p$ coordinate. Mon. Weather Rev. 120, 851-862. 